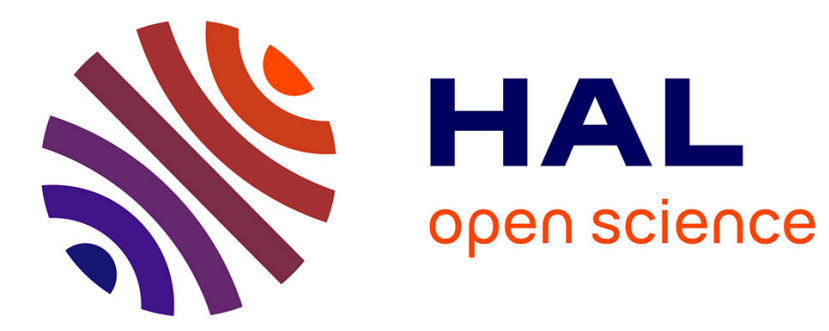

\title{
Dynamics framework for 2D anisotropic continuum-discrete damage model for progressive localized failure of massive structures
}

\author{
Xuan Khanh Do, Adnan Ibrahimbegovic, Delphine Brancherie
}

\section{To cite this version:}

Xuan Khanh Do, Adnan Ibrahimbegovic, Delphine Brancherie. Dynamics framework for 2D anisotropic continuum-discrete damage model for progressive localized failure of massive structures. Computers \& Structures, 2017, 183, pp.14-26. 10.1016/j.compstruc.2017.01.011 . hal-01997373

\section{HAL Id: hal-01997373 \\ https://hal.utc.fr/hal-01997373}

Submitted on 5 Feb 2019

HAL is a multi-disciplinary open access archive for the deposit and dissemination of scientific research documents, whether they are published or not. The documents may come from teaching and research institutions in France or abroad, or from public or private research centers.
L'archive ouverte pluridisciplinaire HAL, est destinée au dépôt et à la diffusion de documents scientifiques de niveau recherche, publiés ou non, émanant des établissements d'enseignement et de recherche français ou étrangers, des laboratoires publics ou privés. 


\title{
Dynamics framework for 2D anisotropic continuum-discrete damage model for progressive localized failure of massive structures
}

\author{
Xuan Nam Do ${ }^{a}$, Adnan Ibrahimbegovic ${ }^{a, b, *}$, Delphine Brancherie ${ }^{a}$ \\ a Sorbonne Universités/Université de Technologie Compiègne, Laboratoire Roberval de Mécanique, Centre de Recherche Royallieu, 60200 Compiègne, France \\ ${ }^{\mathrm{b}}$ Chair for Computational Mechanics \& IUF, France
}

\section{A R T I C L E I N F O}

\section{Article history:}

Received 5 October 2016

Accepted 18 January 2017

Available online 2 February 2017

\section{Keywords:}

Dynamics

Embedded discontinuity

Fracture process zone - FPZ

Localized failure

\begin{abstract}
A B S T R A C T
We propose a dynamics framework for representing progressive localized failure in materials under quasi-static loads. The proposed model exhibits no mesh dependency, since localization phenomena are taken into account by using the embedded strong discontinuities approach. Robust numerical tool for simulation of discontinuities, in which the displacement field is enhanced to capture the discontinuity, is combined with continuum damage representation of FPZ-fracture process zone. Based upon this approach, a two-dimensional finite element model was developed, capable of describing both the diffuse damage mechanism accompanied by initial strain hardening and subsequent softening response of the structure. The results of several numerical simulations, performed on classical mechanical tests under slowly increasing loads such as Brazilian test or three-point bending test were analyzed. The proposed dynamics framework is shown to increase computational robustness. It was found that the final direction of macro-cracks is predicted quite well and that influence of inertia effects on the obtained solutions is fairly modest especially in comparison among different meshes.
\end{abstract}

() 2017 Elsevier Ltd. All rights reserved.

\section{Introduction}

One of the most important reasons that can cause structural failure is material micro-cracking evolving into localized collapse mechanisms (see $[12,25]$ ). The simulation of the behavior of structures and components with discontinuities has become the topic of much interest for the current research in the field of computational mechanics. Several theories have been provided the fundamental foundation for dealing with the simulation of the onset and propagation of cracks in material, both at macroscopic and microscopic levels. Generally speaking, the presently available approaches to model discontinuities can be classified into two main families: the fracture mechanics approach and the continuum mechanics approach. However, it is well documented in $[19,7]$ that using classical continuum mechanics models for post-localization studies where strain-softening phenomena appear is unreliable. Consequently, to overcome the shortcomings of local theories for modeling strain-softening, in the context of continuum mechanics-based

\footnotetext{
* Corresponding author at: Sorbonne Universités/Université de Technologie Compiègne, Laboratoire Roberval de Mécanique, Centre de Recherche Royallieu, 60200 Compiègne, France.

E-mail addresses: xuan-nam.do@utc.fr (X.N. Do), adnan.ibrahimbegovic@utc.fr (A. Ibrahimbegovic), delphine.brancherie@utc.fr (D. Brancherie).
}

models, the embedded discontinuity approach (EDA) was recently introduced giving rise to two variants of weak embedded discontinuity formulations and strong embedded discontinuity formulations. In the former case, with representative works in [22,28], the strain field becomes discontinuous, but the displacement field remaining continuous, across the limits of a narrow band (strain localization band). Alternative approach concerns the case when the strain localization band collapses into a surface, so-called displacement discontinuity. The displacement field that becomes discontinuous across that surface implies that the strain field becomes unbounded (e.g. $[1,2,6,9,11,16,20,24,27,30])$. Yet another alternative method is the extended finite element method (XFEM), in which a global approximation to the strong discontinuity kinematics is supplied by exploiting the partition of unity property of the shape functions (see [8]). In comparison to XFEM, the embedded discontinuity method has more computational advantage. Namely, in the approximation of the displacement field, XFEM requires additional nodal degrees of freedom, while in EDA the additional degree of freedom can be eliminated by static condensation at the element level, so that the dimension of the discretized problem does not increase at global level. As a consequence, for the efficiency reasons, the embedded strong discontinuity method is chosen in this work.

The vast majority of the previous studies using the embedded discontinuity approach only considered quasi-static problems. 
Fairly few works in dynamics were carried out with this approach, such as [13] or [5]. As the main novelty here, we present a twodimensional model with the main contributions as follows:

- Capability of representing the localized failure of massive structure in dynamics by taking into account combination of strain hardening in FPZ-fracture process zone and softening with embedded strong discontinuities.

- Providing an alternative X-FEM approach to modeling failure phenomena in dynamics with a more robust implementation, and a more reliable prediction of final crack direction for massive structures with a significant contribution of FPZ.

- A multi-surface damage model including normal interface and tangential interface damage modes, as generalization of mode I and mode II failure modes in LFM-Linear Fracture Mechanics.

The paper is organized as follows: Section 2 is devoted to the theoretical formulation of the combined continuum damageembedded strong discontinuity model, followed by Section 3 in which the numerical implementation is discussed. In Section 4, we present the results of numerical simulations performed on classical mechanical tests such as Brazilian test or three-point bending test, and analyze. Finally, Section 5 closes the paper with some concluding remarks.

\section{Theoretical formulation}

\subsection{Continuum damage model}

The damage model with isotropic hardening presented herein is based on the idea first proposed in [21] where the internal variable is chosen as the fourth order compliance tensor, $\overline{\mathbf{D}}$ representing an anisotropic damaged state. The damage criterion prescribing the admissible values of stress in the sense of damage is defined:

$\bar{\Phi}(\boldsymbol{\sigma}, \bar{q})=\widehat{\Phi}(\boldsymbol{\sigma})-\left(\bar{\sigma}_{f}-\bar{q}\right) \leqslant 0$

where $\bar{\sigma}_{f}$ refers to the limit of elasticity indicating the first cracking and $\bar{q}$ is a stress-like hardening variable which handles the damage threshold evolution.

The internal energy of such a damage model can formally be written:

$\bar{\chi}(\overline{\boldsymbol{\varepsilon}}, \overline{\mathbf{D}}, \bar{\xi})=\frac{1}{2} \overline{\boldsymbol{\varepsilon}} \bullet \overline{\mathbf{D}}^{-1} \overline{\boldsymbol{\varepsilon}}+\bar{\Xi}(\bar{\xi})$

where $\bar{\xi}$ is the hardening variable, and $\bar{\Xi}(\bar{\xi})$ stands for the corresponding hardening potential.

By using the Legendre transformation to exchange the roles between the stress and deformation, we further introduce the complementary energy potential:

$\bar{\psi}(\boldsymbol{\sigma}, \overline{\mathbf{D}}, \bar{\xi})=\boldsymbol{\sigma} \bullet \overline{\boldsymbol{\varepsilon}}-\bar{\psi}(\overline{\boldsymbol{\varepsilon}}, \overline{\mathbf{D}}, \bar{\xi})=\frac{1}{2} \boldsymbol{\sigma} \bullet \overline{\mathbf{D}} \boldsymbol{\sigma}-\bar{\Xi}(\bar{\xi})$

By exploiting the second law of thermodynamics, we can obtain the explicit form of the damage model dissipation defined as follows:

$0 \leqslant \overline{\mathcal{D}}=\boldsymbol{\sigma} \bullet \dot{\overline{\boldsymbol{\varepsilon}}}-\dot{\bar{\psi}}(\overline{\boldsymbol{\varepsilon}}, \overline{\mathbf{D}}, \bar{\xi})=\dot{\boldsymbol{\sigma}}[-\overline{\boldsymbol{\varepsilon}}+\overline{\mathbf{D}} \boldsymbol{\sigma}]+\frac{1}{2} \boldsymbol{\sigma} \bullet \dot{\overline{\mathbf{D}} \boldsymbol{\sigma}}-\frac{d \bar{\Xi}(\bar{\xi})}{d \bar{\xi}} \dot{\bar{\xi}}$

In the case of "elastic" process where $\dot{\overline{\mathbf{D}}}=0$ and $\dot{\bar{\xi}}=0$, the dissipation inequality (the Clausius-Duhem inequality) above becomes an equality, $\overline{\mathcal{D}}=0$, and leads to the appropriate form of constitutive equations for damage model can be established:

$\overline{\boldsymbol{\varepsilon}}=\overline{\mathbf{D}} \boldsymbol{\sigma} \Rightarrow \boldsymbol{\sigma}=\overline{\mathbf{D}}^{-1} \overline{\boldsymbol{\varepsilon}}=\frac{\partial \bar{\psi}(\overline{\boldsymbol{\varepsilon}}, \overline{\mathbf{D}}, \bar{\xi})}{\partial \overline{\boldsymbol{\varepsilon}}} ; ; \bar{q}=-\frac{d \bar{\Xi}(\bar{\xi})}{d \bar{\xi}}$
By assuming that those results remain valid for an inelastic process in which the internal variables are now modified, $\dot{\overline{\mathbf{D}}} \neq 0 \quad \dot{\bar{\xi}} \neq 0$, we can define the positive damage dissipation:

$0<\overline{\mathcal{D}}=\frac{1}{2} \boldsymbol{\sigma} \bullet \dot{\overline{\mathbf{D}}} \boldsymbol{\sigma}+\overline{\bar{q}} \dot{\bar{\xi}}$

In order to obtain the associated evolution equations for internal variables, the principle of maximum dissipation has to be enforced under the constraints $\bar{\Phi}(\boldsymbol{\sigma}, \bar{q}) \leqslant 0$. By introducing Lagrange multiplier $\bar{\gamma}$, the previous problem can then be recast as the unconstrained maximization problem:

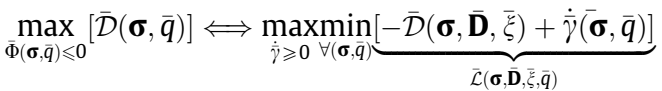

Applying the Kuhn-Tucker optimality conditions for the chosen damage Lagrangian the evolution equations for the internal variables can thus be written:

$0=\frac{\partial \overline{\mathcal{L}}(\boldsymbol{\sigma}, \overline{\mathbf{D}}, \bar{\xi}, \bar{q})}{\partial \boldsymbol{\sigma}} \Rightarrow \dot{\overline{\mathbf{D}}}=\dot{\bar{\gamma}} \frac{\partial \bar{\Phi}(\boldsymbol{\sigma}, \bar{q})}{\partial \boldsymbol{\sigma}} \otimes \frac{1}{\boldsymbol{\sigma}}$

$0=\frac{\partial \overline{\mathcal{L}}(\boldsymbol{\sigma}, \overline{\mathbf{D}}, \bar{\xi}, \bar{q})}{\partial \bar{q}} \Rightarrow \dot{\bar{\xi}}=\dot{\bar{\gamma}} \frac{\partial \bar{\Phi}(\boldsymbol{\sigma}, \bar{q})}{\partial \bar{q}}$

Since $\widehat{\bar{\Phi}}(\boldsymbol{\sigma})$ is a homogeneous function of degree one, which implies that $\frac{\partial \bar{\Phi}}{\partial \boldsymbol{\sigma}} \boldsymbol{\sigma}=\widehat{\bar{\Phi}}(\boldsymbol{\sigma})$, from (8) we can write the evolution of damage model compliance as:

$\dot{\overline{\mathbf{D}}}=\dot{\bar{\gamma}} \frac{\partial \bar{\Phi}}{\partial \boldsymbol{\sigma}} \otimes \frac{\partial \bar{\Phi}}{\partial \boldsymbol{\sigma}} \frac{1}{\widehat{\widehat{\Phi}}(\boldsymbol{\sigma})}$

The corresponding loading-unloading criteria are also a part the Kuhn-Tucker optimality conditions:

$\dot{\bar{\gamma}} \geqslant 0, \bar{\Phi}(\boldsymbol{\sigma}, \bar{q}) \leqslant 0, \dot{\bar{\gamma}} \bar{\Phi}(\boldsymbol{\sigma}, \bar{q})=0$

Finally, the consistency condition which takes form as $\dot{\bar{\gamma}} \dot{\bar{\Phi}}(\boldsymbol{\sigma}, \bar{q})=0$, will lead to the corresponding value of damage multiplier.

$\dot{\bar{\gamma}}=\frac{\frac{\partial \bar{\Phi}}{\partial \boldsymbol{\sigma}} \bullet \overline{\mathbf{D}}^{-\mathbf{1}} \dot{\overline{\boldsymbol{\varepsilon}}}}{\frac{\partial \bar{\Phi}}{\partial \boldsymbol{\sigma}} \bullet \overline{\mathbf{D}}^{-1} \frac{\partial \bar{\Phi}}{\partial \boldsymbol{\sigma}}+\bar{K}\left(\frac{\partial \bar{\Phi}}{\partial \bar{q}}\right)^{2}}$

where $\bar{K}$ is the hardening modulus for the bulk material.

With these results in hand, we can easily write the stress rate constitutive equations for the continuum damage model (see Fig. 1):

$\dot{\boldsymbol{\sigma}}= \begin{cases}\overline{\mathbf{D}}^{-1} \dot{\overline{\boldsymbol{\varepsilon}}} & \dot{\bar{\gamma}}=0 \\ {\left[\overline{\mathbf{D}}^{-1}-\frac{\left(\overline{\mathbf{D}}^{-1} \frac{\partial \bar{\Phi}}{\partial \mathbf{\sigma}}\right) \otimes\left(\overline{\mathbf{D}}^{-1} \frac{\partial \bar{\Phi}}{\partial \boldsymbol{\sigma}}\right)}{\frac{\partial \bar{\Phi}}{\partial \mathbf{\sigma}} \bullet \overline{\mathbf{D}}^{-1} \frac{\partial \bar{\Phi}}{\partial \boldsymbol{\sigma}}+\bar{K}\left(\frac{\partial \bar{\Phi}}{\partial \bar{q}}\right)^{2}}\right] \dot{\overline{\boldsymbol{\varepsilon}}}} & \dot{\bar{\gamma}}>0\end{cases}$

\subsection{Discrete damage model}

The damage model of this kind is further enhanced to be able to describe localized failure leading to softening. The localized failure is represented by a strong discontinuity in the displacement field across the surface $\Gamma_{s}$ (see Fig. 2). Therefore, the total displacement field can be written as the sum of a continuous regular part $\overline{\mathbf{u}}(\mathbf{x}, t)$ and a discontinuous irregular part corresponding to the displacement jump $\overline{\mathbf{u}}(\mathbf{x}, t)$ (see also [31] and [32]) (see Fig. 3):

$\mathbf{u}(\mathbf{x}, t)=\overline{\mathbf{u}}(\mathbf{x}, t)+\overline{\overline{\mathbf{u}}}(\mathbf{x}, t) H_{\Gamma_{s}}(\mathbf{x})$

where $H_{\Gamma_{s}}(\mathbf{x})$ denotes the Heaviside function (see Fig. 4):

$H_{\Gamma_{s}}(\mathbf{x})= \begin{cases}1 & \mathbf{x} \in \partial \Omega^{-} \\ 0 & \mathbf{x} \in \partial \Omega^{+}\end{cases}$ 

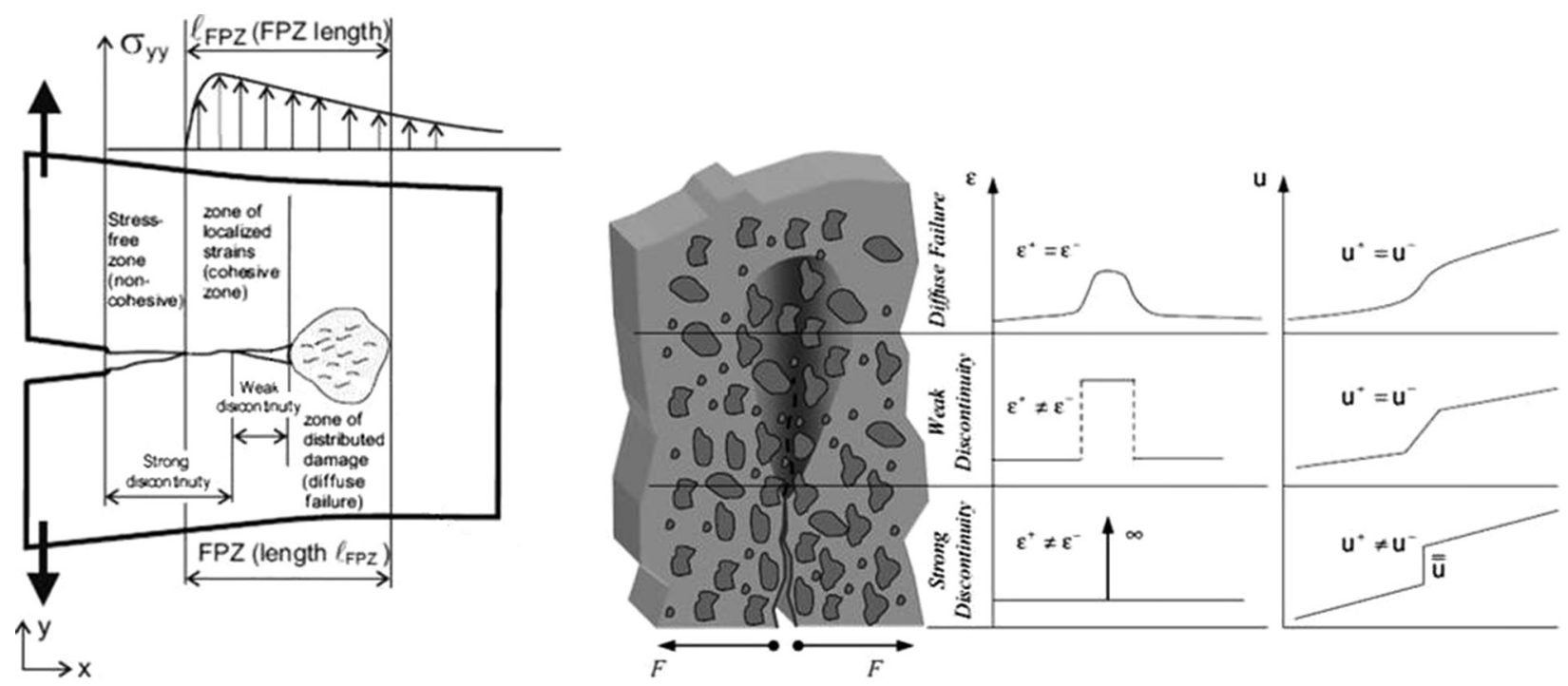

Fig. 1. Kinematics in the fracture process zone.
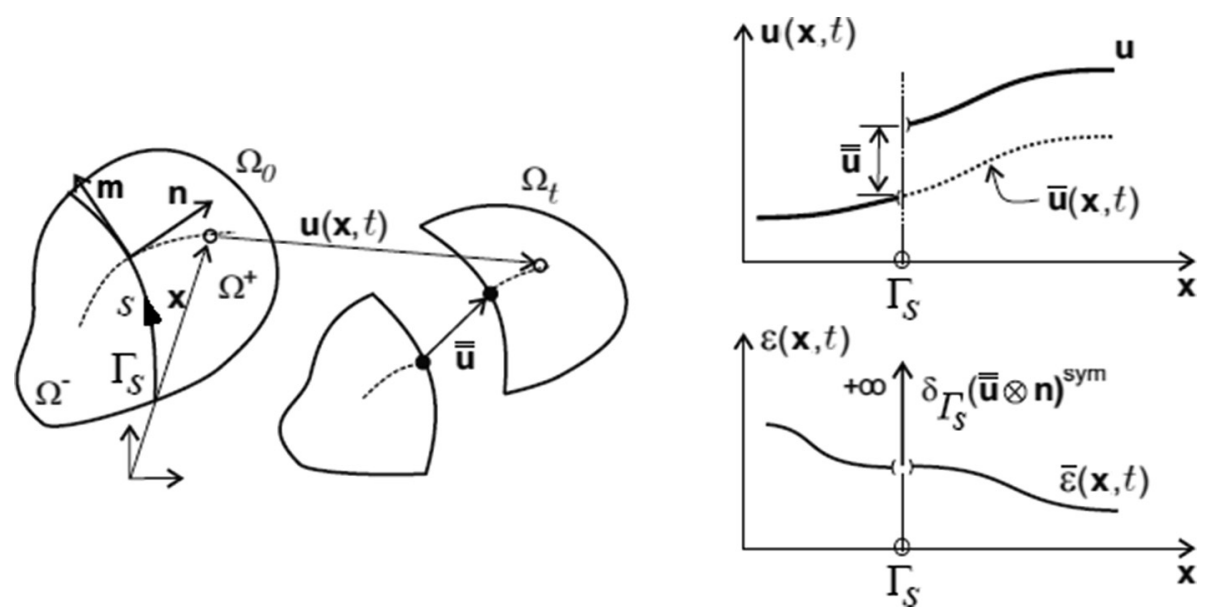

Fig. 2. The discontinuity surface $\Gamma_{s}$ separating the domain $\Omega$ into $\Omega^{+}$and $\Omega^{-}$and strong discontinuity kinematics.

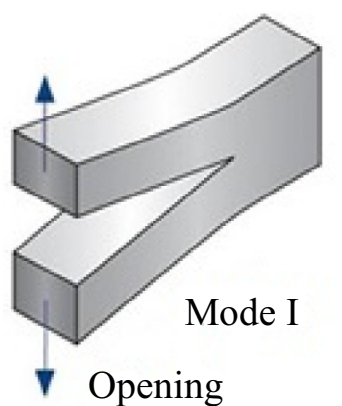

Fig. 3. Fracture modes of a $2 \mathrm{D}$ anisotropic damage model.

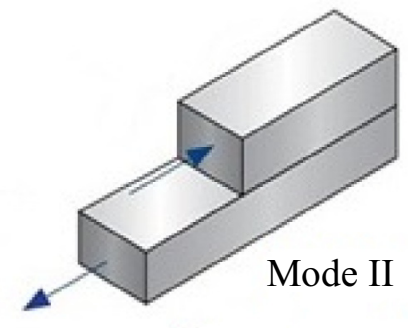

Sliding

with $\partial \Omega^{-}$and $\partial \Omega^{+}$are the boundary of two sub-domains of the element separated by the discontinuity

$\partial \boldsymbol{\Omega}^{-}=\partial \boldsymbol{\Omega} \cap \boldsymbol{\Omega}^{-} \quad \partial \boldsymbol{\Omega}^{+}=\partial \boldsymbol{\Omega} \cap \boldsymbol{\Omega}^{+}$

and $\Gamma_{s}$ is the discontinuity surface separating the continuous domain $\Omega$ into sub-domains $\Omega^{+}$and $\Omega^{-}$.

The infinitesimal strain which corresponds to this displacement decomposition can be then computed as the sum of a regular (continuous) part, $\overline{\boldsymbol{\varepsilon}}(\mathbf{x}, t)$, and a singular (discontinuous) part, $\overline{\bar{\varepsilon}}(\mathbf{x}, t)$, according to:

$\boldsymbol{\varepsilon}(\mathbf{x}, t)=\overline{\mathbf{\varepsilon}}(\mathbf{x}, t)+\overline{\overline{\mathbf{\varepsilon}}}(\mathbf{x}, t) \delta_{\Gamma_{s}}(\mathbf{x})$

where
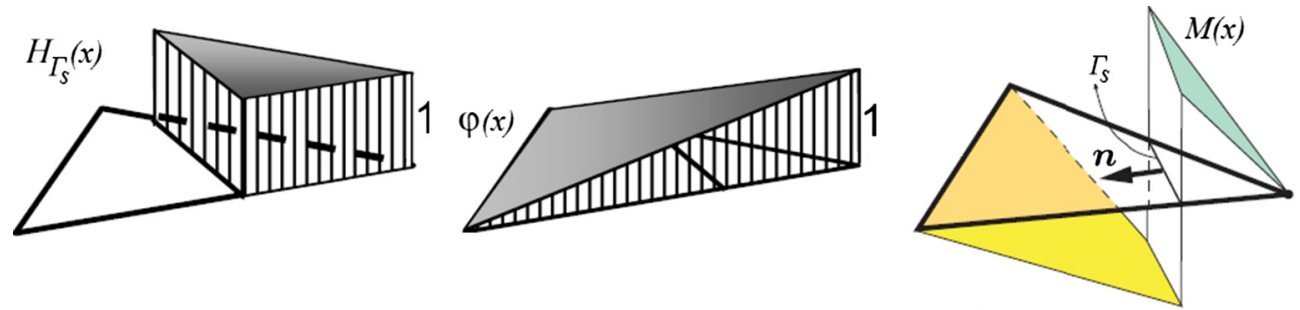

Fig. 4. Discontinuous shape function (2D case) for a CST element with constant discontinuity jumps. 
$\overline{\mathbf{\varepsilon}}(\mathbf{x}, t)=\nabla^{s} \overline{\mathbf{u}}(\mathbf{x}, t)+H_{\Gamma_{s}} \nabla^{s} \overline{\overline{\mathbf{u}}}(\mathbf{x}, t)$

$\overline{\overline{\mathbf{\varepsilon}}}(\mathbf{x}, t)=(\overline{\overline{\mathbf{u}}}(\mathbf{x}, t) \otimes \mathbf{n})^{s}$

From Eq. (5), the strain field can be written in terms of the stress field. By taking into account that the stress field remains bounded, we can conclude that the damage compliance tensor $\mathbf{D}$ should also be split into two parts: regular and singular:

$\mathbf{D}=\overline{\mathbf{D}}+\overline{\overline{\mathbf{D}}} \delta_{\Gamma_{s}}$

Combining Eqs. (15)-(17), we can identify:

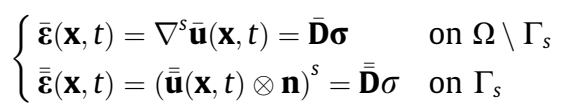

The decomposition of the strain field into a regular part and singular part leads to the corresponding split of hardening variable $\xi$ so that $\xi=\bar{\xi}+\bar{\xi} \delta_{\Gamma_{\mathrm{s}}}$. Deriving from these results, we can write the Helmholtz free energy which is also divided into a regular part $\bar{\psi}$ from fracture process zone on $\backslash \Gamma_{s}$ and a singular part $\bar{\psi}$ associated to the discontinuity on $\Gamma_{s}$ :

$\psi(\boldsymbol{\varepsilon}, \mathbf{D}, \xi)=\underbrace{\frac{1}{2} \overline{\boldsymbol{\varepsilon}} \bullet \overline{\mathbf{D}}^{-1} \overline{\boldsymbol{\varepsilon}}+\bar{\Xi}(\bar{\xi})}_{\bar{\psi}(\overline{\boldsymbol{\varepsilon}}, \overline{\mathbf{D}}, \bar{\xi})}+\underbrace{\left[\frac{1}{2} \overline{\overline{\mathbf{u}}} \bullet \overline{\overline{\mathbf{Q}}}^{-1} \overline{\overline{\mathbf{u}}}+\overline{\bar{\Xi}}(\overline{\bar{\xi}})\right]}_{\overline{\bar{\psi}}(\overline{\overline{\mathbf{u}}}, \overline{\mathbf{Q}}, \overline{\bar{\xi}})} \delta_{\Gamma_{s}}$

where $\overline{\overline{\mathbf{Q}}}=(\mathbf{n} \bullet \overline{\overline{\mathbf{D}}} \mathbf{n})^{-1}$ is internal variable for describing the damage response at the discontinuity.

The total dissipation of the material can then be expressed as the sum of the bulk dissipation due to diffuse damage mechanisms and the localized dissipation due to the development of localization zones:

$$
\begin{aligned}
\mathcal{D} & =\overline{\mathcal{D}}+\overline{\overline{\mathcal{D}}} \delta_{\Gamma_{s}} \\
& =\boldsymbol{\sigma} \bullet \dot{\bar{\varepsilon}}-\frac{d}{d t} \bar{\psi}(\bar{\varepsilon}, \overline{\mathbf{D}}, \bar{\xi})+\left[\mathbf{t}_{\Gamma_{s}} \bullet \dot{\overline{\mathbf{u}}}-\frac{d}{d t} \bar{\psi}(\overline{\overline{\mathbf{u}}}, \overline{\overline{\mathbf{Q}}}, \overline{\bar{\xi}})\right] \delta_{\Gamma_{s}}
\end{aligned}
$$

where the second term is the singular part of dissipation, which can be written:

$0 \leqslant \overline{\overline{\mathcal{D}}}=\dot{\overline{\mathbf{u}}}\left(\mathbf{t}_{\Gamma_{s}}-\overline{\mathbf{Q}}^{-1} \overline{\overline{\mathbf{u}}}\right)+\frac{1}{2} \mathbf{t}_{\Gamma_{s}} \bullet \dot{\overline{\overline{\mathbf{Q}}}} \mathbf{t}_{\Gamma_{s}}-\frac{d \overline{\bar{\Xi}}(\overline{\bar{\xi}})}{d \overline{\bar{\xi}}} \overline{\overline{\bar{\xi}}}$

Each damage dissipation mechanism activation is controlled by the corresponding damage criterion. For the surface of discontinuity, we assume the damage function as:

$\overline{\bar{\Phi}}\left(\mathbf{t}_{\Gamma_{s}}, \overline{\bar{q}}\right)=\widehat{\overline{\bar{\Phi}}}\left(\mathbf{t}_{\Gamma_{s}}\right)-\left(\overline{\bar{\sigma}}_{f}-\overline{\bar{q}}\right) \leqslant 0$

where $\mathbf{t}_{\Gamma_{s}}=\left.(\boldsymbol{\sigma n})\right|_{\Gamma_{s}}$ is the traction vector acting on discontinuity, $\widehat{\bar{\Phi}}\left(\mathbf{t}_{\Gamma_{s}}\right)$ is a homogeneous function of degree one, i.e., $\frac{\partial \overline{\bar{\Phi}}}{\partial \mathbf{t}_{\Gamma_{s}}} \mathbf{t}_{\Gamma_{s}}=\frac{\partial \hat{\overline{\bar{\Phi}}}}{\partial \mathbf{t}_{\Gamma_{s}}} \mathbf{t}_{\Gamma_{s}}=\widehat{\overline{\bar{\Phi}}}\left(\mathbf{t}_{\Gamma_{s}}\right), \overline{\bar{\sigma}}_{f}$ is the initial damage threshold and $\overline{\bar{q}}$ is the softening traction-like variable controlling the evolution of the damage threshold.

In an elastic process, with no change of internal variables and zero dissipation ( $\dot{\overline{\overline{\mathbf{Q}}}}=0, \dot{\overline{\bar{\xi}}}=0, \overline{\overline{\mathcal{D}}}=0$ ), Eq. (21) allows us to define the form of constitutive equation and the traction-like variable associated to softening phenomena at the discontinuity:

$\mathbf{t}_{\Gamma_{s}}=\overline{\overline{\mathbf{Q}}}^{-1} \overline{\overline{\mathbf{u}}}=\frac{\partial \overline{\bar{\psi}}(\overline{\overline{\mathbf{u}}}, \overline{\overline{\mathbf{Q}}}, \overline{\bar{\xi}})}{\partial \overline{\overline{\mathbf{u}}}} ; \quad \overline{\bar{q}}=-\frac{d \overline{\bar{\Xi}}(\overline{\bar{\xi}})}{d \overline{\bar{\xi}}}$

By assuming that these relations also hold in damage process, from Eq. (21) we can obtain a reduced form of the inelastic localized dissipation as:

$\overline{\overline{\mathcal{D}}}=\frac{1}{2} \mathbf{t}_{\Gamma_{s}} \bullet \dot{\overline{\mathbf{Q}}} \mathbf{t}_{\Gamma_{s}}+\overline{\bar{q}} \dot{\overline{\bar{\xi}}}$
Using the principle of maximum of damage dissipation we can choose the traction which will maximize the damage dissipation among all admissible candidates in the sense of the chosen damage criterion:

$\max _{\overline{\bar{\Phi}}\left(\mathbf{t}_{\Gamma_{s}}, \overline{\bar{q}}\right) \leqslant 0}\left[\overline{\overline{\mathcal{D}}}\left(\mathbf{t}_{\Gamma_{s}}, \overline{\bar{q}}\right)\right] \Longleftrightarrow \max _{\overline{\bar{\gamma}} \geqslant 0} \min _{\forall\left(\mathbf{t}_{\Gamma_{s}}, \overline{\bar{q}}\right)}[\underbrace{\left.-\overline{\overline{\mathcal{D}}}\left(\mathbf{t}_{\Gamma_{s}}, \overline{\overline{\mathbf{Q}}}, \overline{\bar{\xi}}\right)+\dot{\overline{\bar{\Phi}}} \overline{\bar{\Phi}}\left(\mathbf{t}_{\Gamma_{s}}, \overline{\bar{q}}\right)\right]}_{\overline{\overline{\mathcal{L}}}\left(\mathbf{t}_{\Gamma_{s}}, \overline{\overline{\mathbf{Q}}, \overline{\overline{\mathcal{D}}}, \overline{\bar{q}})}\right.}$

where $\overline{\bar{\gamma}}$ stands for the Lagrange multiplier introduced for the discontinuity.

Combining the last result and the corresponding Kuhn-Tucker optimality conditions for maximization problem in Eq. (25), it is possible to provide the evolution equations for the internal variables:

$0=\frac{\partial \overline{\overline{\mathcal{L}}}\left(\mathbf{t}_{\Gamma_{s}}, \overline{\bar{Q}}, \overline{\bar{\xi}}, \overline{\bar{q}}\right)}{\partial \mathbf{t}_{\Gamma_{s}}} \Rightarrow \dot{\overline{\overline{\mathbf{Q}}}}=\dot{\overline{\bar{\gamma}}} \frac{\partial \overline{\bar{\Phi}}\left(\mathbf{t}_{\Gamma_{s}}, \overline{\bar{q}}\right)}{\mathbf{t}_{\Gamma_{s}}} \otimes \frac{1}{\mathbf{t}_{\Gamma_{s}}}$

$0=\frac{\partial \overline{\overline{\mathcal{L}}}\left(\mathbf{t}_{\Gamma_{s}}, \overline{\bar{Q}}, \overline{\bar{\xi}}, \overline{\bar{q}}\right)}{\partial \overline{\bar{q}}} \Rightarrow \dot{\overline{\bar{\xi}}}=\dot{\overline{\bar{\gamma}}} \frac{\partial \overline{\bar{\Phi}}\left(\mathbf{t}_{\Gamma_{s}}, \overline{\bar{q}}\right)}{\partial \overline{\bar{q}}}$

The homogeneity of function $\widehat{\overline{\bar{\Phi}}}(\boldsymbol{\sigma})$ allows us to obtain the final form of the evolution of damage model compliance as:

$\dot{\overline{\mathbf{Q}}}=\dot{\overline{\bar{\gamma}}} \frac{1}{\overline{\bar{\Phi}}\left(\mathbf{t}_{\Gamma_{s}}\right)} \frac{\partial \overline{\bar{\Phi}}}{\partial \mathbf{t}_{\Gamma_{s}}} \otimes \frac{\partial \overline{\bar{\Phi}}}{\partial \mathbf{t}_{\Gamma_{s}}}$

These equations are accompanied by loading-unloading conditions:

$\dot{\bar{\gamma}} \geqslant 0 \overline{\bar{\Phi}}\left(\mathbf{t}_{\Gamma_{s}}, \overline{\bar{q}}\right) \leqslant 0 \dot{\bar{\gamma}} \overline{\bar{\Phi}}\left(\mathbf{t}_{\Gamma_{s}}, \overline{\bar{q}}\right)=0$

Finally, we get the consistency condition which is written as:

$\dot{\bar{\gamma}} \dot{\bar{\Phi}}\left(\mathbf{t}_{\Gamma_{s}}, \overline{\bar{q}}\right)=0$

The Lagrange multiplier value for the damage step can easily be computed as follows:

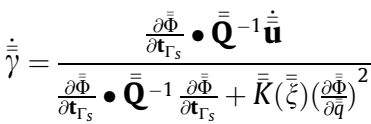

where $\overline{\bar{K}}$ is the softening modulus.

From Eqs. (26) and (30) we can obtain the rate constitutive equations between traction and "jump" in displacement, according to:

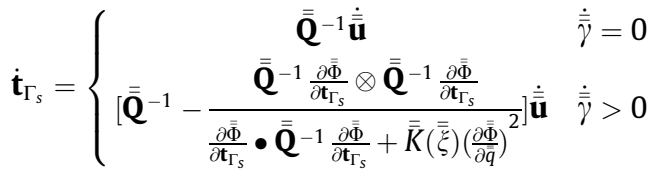

\subsection{Choice of damage criteria}

The isotropic damage criterion defining the elastic domain is chosen as (see [18]):

$\bar{\Phi}(\boldsymbol{\sigma}, \bar{q})=\sqrt{\boldsymbol{\sigma} \bullet \mathbf{D}^{\mathrm{e}} \boldsymbol{\sigma}}-\frac{1}{\sqrt{E}}\left(\bar{\sigma}_{f}-\bar{q}\right)$

where $\mathbf{D}^{\mathrm{e}}$ denotes the undamaged elastic compliance tensor of the bulk material, which is equal to the inverse of elasticity tensor, $\mathbf{D}^{\mathrm{e}}=\left[\mathbf{C}^{e}\right]^{-1}$, and $E$ refers to the Young modulus. From there, we get:

$\frac{\partial \bar{\Phi}}{\partial \boldsymbol{\sigma}}=\frac{\mathbf{D}^{e} \boldsymbol{\sigma}}{\|\boldsymbol{\sigma}\|_{\mathbf{D}^{e}}} ; \frac{\partial \bar{\Phi}}{\partial \bar{q}}=\frac{1}{\sqrt{E}}$

where $\|\boldsymbol{\sigma}\|_{\mathbf{D}^{e}}=\sqrt{\boldsymbol{\sigma} \bullet \mathbf{D}^{e} \boldsymbol{\sigma}}$ defines the corresponding norm in the stress space. 
Combining Eqs. (8) and (33), we can thus write the evolution equations of internal variables in a simplified form as:

$\left\{\begin{array}{l}\dot{\overline{\mathbf{D}}}=\dot{\bar{\gamma}} \frac{\mathbf{D}^{e}}{\|\boldsymbol{\sigma}\|_{\mathbf{D}^{e}}} \\ \dot{\bar{\xi}}=\dot{\bar{\gamma}} \frac{1}{\sqrt{E}}\end{array}\right.$

To describe a particular damage mechanism of a multi-surface model each damage surface $\overline{\bar{\Phi}}_{k}\left(\mathbf{t}_{\Gamma_{\mathrm{s}}}, \overline{\bar{q}}\right)$ is chosen, according to:

$\overline{\bar{\Phi}}_{k}\left(\mathbf{t}_{\Gamma_{\mathrm{s}}}, \overline{\bar{q}}\right) \leqslant 0 \quad k=1,2, \ldots, m$

In that way, for a 2D anisotropic damage model (two-surface damage model) which makes use of the crack opening displacement $\overline{\mathbf{u}}_{\mathrm{n}}$ corresponding to mode I in traction and the sliding displacement over the crack mouth $\overline{\overline{\mathbf{u}}}_{\mathrm{m}}$ related to mode II in shear (also see [4]), we can thus write:

$$
\begin{aligned}
& \overline{\bar{\Phi}}_{1}\left(\mathbf{t}_{\Gamma_{s}}, \overline{\bar{q}}\right)=n \bullet \underbrace{\boldsymbol{\sigma n}}_{\mathbf{t}_{\Gamma_{s}}}-\left(\overline{\bar{\sigma}}_{f}-\overline{\bar{q}}\right)=\underbrace{\mathbf{n} \bullet \mathbf{t}_{\Gamma_{s}}}_{\hat{\bar{\Phi}}_{1}\left(\mathbf{t}_{\Gamma_{s}}\right)}-\left(\overline{\bar{\sigma}}_{f}-\overline{\bar{q}}\right) \leqslant 0 \\
& \overline{\bar{\Phi}}_{2}\left(\mathbf{t}_{\Gamma_{s}}, \overline{\bar{q}}\right)=m \bullet \underbrace{\boldsymbol{\sigma n}}_{\mathbf{t}_{\Gamma_{s}}}-\left(\overline{\bar{\sigma}}_{s}-\frac{\overline{\bar{\sigma}}_{s}}{\overline{\bar{\sigma}}_{f}} \overline{\bar{q}}\right)=\underbrace{\left|\mathbf{m} \bullet \mathbf{t}_{\Gamma_{s}}\right|}_{\widehat{\bar{\Phi}}_{2}\left(\mathbf{t}_{\Gamma_{s}}\right)}-\left(\overline{\bar{\sigma}}_{s}-\frac{\overline{\bar{\sigma}}_{s}}{\overline{\bar{\sigma}}_{f}} \overline{\bar{q}}\right) \leqslant 0
\end{aligned}
$$

where $\mathbf{n}, \mathbf{m}$ are respectively the external unit normal and tangent vectors on the crack, $\overline{\bar{\sigma}}_{f}$ is the given fracture stress, $\overline{\bar{\sigma}}_{s}$ is the limit value of shear stress on the discontinuity and $\overline{\bar{q}}$ is the softening traction-like variable through which the two failure functions above are coupled.

For representing the final fracture phase, we chose an exponential softening law, according to:

$\overline{\bar{q}}=\overline{\bar{\sigma}}_{f}\left[1-\exp \left(-\frac{\overline{\bar{\beta}}}{\overline{\bar{\sigma}}} \overline{\bar{\xi}}\right)\right] \Rightarrow \overline{\bar{K}}=-\frac{\partial \overline{\bar{q}}}{\partial \overline{\bar{\xi}}}=-\overline{\bar{\beta}} \exp \left(-\frac{\overline{\bar{\beta}}}{\overline{\bar{\sigma}}} \overline{\bar{\xi}}\right)$

where $\overline{\bar{\beta}}$ is a parameter chosen in accordance with the fracture energy dissipated at the discontinuity. By integrating the total dissipation along the fracture process, we obtain the fracture energy:

$G_{f}=\int_{0}^{\infty} \overline{\bar{\sigma}}_{f} \exp \left(-\frac{\overline{\bar{\beta}}}{\overline{\bar{\sigma}}} \overline{\bar{\xi}}\right) d \overline{\bar{\xi}}$

The evolution equations for internal variables:

$\dot{\overline{\mathbf{Q}}}=\dot{\overline{\bar{\gamma}}}_{1} \frac{1}{\mathbf{n} \bullet \mathbf{t}_{\Gamma_{s}}} \boldsymbol{n} \otimes n+\dot{\overline{\bar{\gamma}}}_{2} \frac{1}{\left|\mathbf{m} \bullet \mathbf{t}_{\Gamma_{s}}\right|} m \otimes m$

$\dot{\overline{\bar{\xi}}}=\dot{\overline{\bar{\gamma}}}_{1} \frac{\partial \overline{\bar{\Phi}}_{1}}{\partial \overline{\bar{q}}}+\dot{\overline{\bar{\gamma}}}_{2} \frac{\partial \overline{\bar{\Phi}}_{2}}{\partial \overline{\bar{q}}}=\dot{\overline{\bar{\gamma}}}_{1}+\dot{\overline{\bar{\gamma}}}_{2} \frac{\overline{\bar{\sigma}}_{s}}{\overline{\bar{\sigma}}_{f}}$

From the consistency conditions $\dot{\overline{\bar{\gamma}}}_{i} \dot{\bar{\Phi}}_{1}\left(\mathbf{t}_{\Gamma_{s}}, \overline{\bar{q}}\right)=0 ; i=1$, 2 , we can obtain the value of Lagrange multipliers as:

$\dot{\overline{\bar{\gamma}}}_{i}=\sum_{j=1}^{2} \mathbf{G}_{i j}^{-1} \frac{\partial \overline{\bar{\Phi}}_{i}}{\partial \mathbf{t}_{\Gamma_{s}}} \bullet \overline{\overline{\mathbf{Q}}}-1 \dot{\overline{\mathbf{u}}} ; i=1,2$

where

$$
\begin{aligned}
& \mathbf{G}=\left[\begin{array}{cc}
\mathbf{n} \bullet \overline{\overline{\mathbf{Q}}}^{-1} \mathbf{n}+\overline{\overline{\mathbf{K}}}(\overline{\bar{\xi}}) & \overline{\overline{\mathbf{K}}}(\overline{\bar{\xi}}) \frac{\overline{\bar{\sigma}}_{s}}{\bar{\sigma}_{f}} \\
\overline{\overline{\mathbf{K}}}(\overline{\bar{\xi}}) \overline{\bar{\sigma}}_{s} & \mathbf{m} \bullet \overline{\overline{\mathbf{Q}}}^{-1} \mathbf{m}+\overline{\overline{\mathbf{K}}}(\overline{\bar{\xi}})\left(\overline{\bar{\sigma}_{s}}\right)^{2}
\end{array}\right] ; \\
& \overline{\overline{\mathbf{Q}}}=\left[\begin{array}{cc}
\overline{\bar{Q}}_{n n} & 0 \\
0 & \overline{\bar{Q}}_{m m}
\end{array}\right]_{(n, m)}
\end{aligned}
$$

With these results in hand, the stress rate constitutive equations for discrete damage model can easily be written as follows:

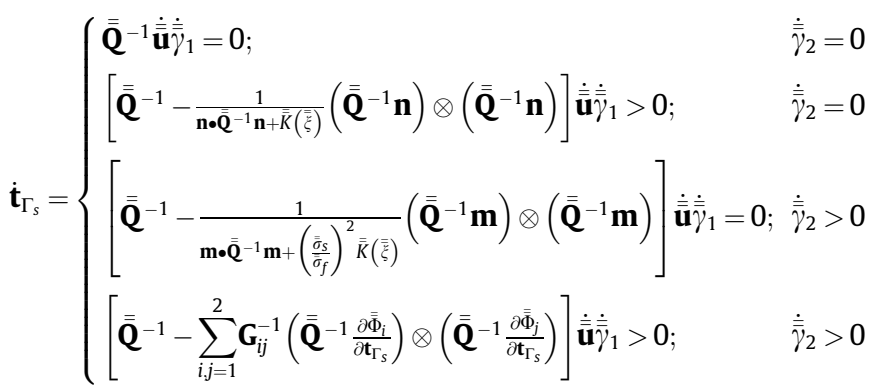

\section{Numerical implementation - Finite element with embedded strong discontinuities}

\subsection{Enhanced kinematics}

As stated earlier, once the failure in a local zone occurs, the enhanced displacement field ought to be introduced and written as the sum of a regular part and an irregular part (see Fig. 2). In this direction, we present herein the finite element interpolations for a triangular three-node element (CST) in which the displacement jump is taken as constant.

Thus, the total displacement field $\mathbf{u}(\mathbf{x}, t)$ can be written as:

$\mathbf{u}(\mathbf{x}, t)=\hat{\mathbf{u}}(\mathbf{x}, t)+\overline{\overline{\mathbf{u}}}(\mathbf{x}, \mathrm{t})\left[H_{\Gamma_{s}}(\mathbf{x})-\varphi(\mathbf{x})\right]$

where $\hat{\mathbf{u}}(\mathbf{x}, t)$ is the classic displacement interpolation of a CST finite element from which we can get the regular strain field:

$\hat{\mathbf{u}}(\mathbf{x}, t)=\sum_{a=1}^{3} \mathbf{N}_{a}(\mathbf{x}) \mathbf{u}_{a}=\mathbf{N d} \Rightarrow \hat{\varepsilon}(\mathbf{x}, t)=\sum_{a=1}^{3} \underbrace{\mathbf{L} \mathbf{N}_{a}(\mathbf{x})}_{\mathbf{B}_{a}(\mathbf{x})} \mathbf{u}_{a}=\mathbf{B d}$

in which $\mathbf{u}_{a}$ refers to the displacement of node $a, \mathbf{N}_{a}(\mathbf{x})$ stands for the shape function associated to node $a$ and $\mathbf{L}$ denotes the matrix form of the strain-displacement operator $\nabla^{s}$. By introducing an additional shape function $\mathbf{M}(\mathbf{x})=H_{\Gamma_{s}}(\mathbf{x})-\varphi(\mathbf{x})$ shown in Fig. 4, the following approximation is considered for the enhanced displacement field:

$\overline{\overline{\mathbf{u}}}(\mathbf{x}, t)=\mathbf{M} \overline{\overline{\mathbf{u}}}$

The real strain field interpolation remains similar to the interpolation of virtual strain field:

$\boldsymbol{\varepsilon}(\mathbf{x}, t)=\mathbf{B d}+\mathbf{G}_{\mathbf{r}} \overline{\overline{\mathbf{u}}} \Rightarrow \delta \boldsymbol{\varepsilon}(\mathbf{x}, t)=\mathbf{B w}+\mathbf{G}_{\mathbf{v}} \overline{\bar{\beta}}$

where $\mathbf{B}_{a}(\mathbf{x})=\mathbf{L N}_{a}(\mathbf{x}), \mathbf{G}_{\mathbf{r}}(\mathbf{x})=\mathbf{L M}(\mathbf{x})$, w and $\overline{\bar{\beta}}$ represent the virtual displacement and virtual displacement jump fields, respectively. $\mathbf{G}_{\mathbf{v}}(\mathbf{x})$ is referred to as an incompatible mode function modified in order to satisfy the patch-test condition. In concordance with the form of the function $\mathbf{M}(\mathbf{x}), \mathbf{G}_{\mathbf{r}}(\mathbf{x})$ and $\mathbf{G}_{\mathbf{v}}(\mathbf{x})$ must be decomposed into a regular part and a singular part as:

$$
\begin{aligned}
& \mathbf{G}_{\mathbf{r}}(\mathbf{x})=\overline{\mathbf{G}}_{\mathbf{r}}(\mathbf{x})+\overline{\overline{\mathbf{G}}}_{\mathbf{r}}(\mathbf{x}) \delta_{\Gamma_{s}} \\
& \mathbf{G}_{\mathbf{v}}(\mathbf{x})=\mathbf{G}_{\mathbf{r}}(\mathbf{x})-\frac{1}{A^{e}} \int_{\Omega^{e}} \mathbf{G}_{\mathbf{r}}(\mathbf{x}) d \Omega^{e}=\overline{\mathbf{G}}_{\mathbf{v}}+\overline{\overline{\mathbf{G}}}_{\mathbf{v}} \delta_{\Gamma_{s}}
\end{aligned}
$$

\subsection{Computational procedure}

The solution of the problem is computed by the operator split solution procedure (see [15]). The global phase will provide the best iterative value of the total strain field, together with the corresponding iterative value of the crack opening and sliding. However, before the global computation can go on, we need to carry out the local computations for the values of the tangent elastodam- 
age modulus $\left(\mathbf{C}^{e d}\right)$ and stress update at the element level (Gauss quadrature point). This is done by using the implicit backward Euler scheme to integrate the rate constitutive equations. The local computation is started by considering the elastic trial state with no evolution of internal variables at time step $t_{n+1}$, namely:

$$
\begin{aligned}
\bar{\gamma}_{n+1} & =0, \bar{\xi}_{n+1}^{\text {trial }}=\bar{\xi}_{n}, \overline{\mathbf{D}}_{n+1}^{\text {trial }}=\overline{\mathbf{D}}_{n}, \bar{q}_{n+1}^{\text {trial }}=\bar{q}_{n}, \boldsymbol{\sigma}_{n+1}^{\text {trial }}=\overline{\mathbf{D}}_{n}^{-1} \overline{\boldsymbol{\varepsilon}}_{n+1} \\
& \Rightarrow \bar{\Phi}_{n+1}^{\text {trial }}=\left\|\boldsymbol{\sigma}_{n+1}^{\text {trial }}\right\|_{\mathbf{D}^{e}}-\frac{1}{\sqrt{E}}\left(\bar{\sigma}_{f}-\bar{q}_{n}\right)
\end{aligned}
$$

If such a damage function has non-positive value, the trial step solution can be accepted as final. On the contrary, if any value the damage function takes is larger than zero the true positive value of $\bar{\gamma}_{n+1}$ and the final values of internal variables must be computed so that $\bar{\Phi}_{n+1}=0$, according to:

$$
\left\{\begin{array}{l}
\overline{\mathbf{D}}_{n+1}=\overline{\mathbf{D}}_{n}+\bar{\gamma}_{n+1} \frac{\mathbf{D}^{e}}{\left\|\boldsymbol{\sigma}_{n+1}\right\|_{\mathbf{D}^{e}}} \\
\bar{\xi}_{n+1}=\bar{\xi}_{n}+\bar{\gamma}_{n+1} \frac{1}{\sqrt{E}}
\end{array}\right.
$$

Exploiting equation $\bar{\Phi}_{n+1}\left(\boldsymbol{\sigma}_{n+1}, \bar{q}_{n+1}\right)=0, \bar{\gamma}_{n+1}$ can be computed as follows:

$\bar{\Phi}_{n+1}=\bar{\Phi}_{n+1}^{\text {trial }}-\frac{\bar{\gamma}_{n+1}}{1+\bar{\mu}_{n}}+\frac{\bar{K}}{E} \bar{\gamma}_{n+1}=0 \Rightarrow \bar{\gamma}_{n+1}=\frac{\bar{\Phi}_{n+1}^{\text {trial }}}{\frac{1}{1+\bar{\mu}_{n}}+\frac{\bar{K}}{E}}$

The last result allows us to obtain the consistent elastodamage tangent modulus, $\mathbf{C}_{n+1}^{e d}=\frac{\partial \boldsymbol{\sigma}_{n+1}}{\partial \bar{\varepsilon}_{n+1}}$

$$
\begin{aligned}
\mathbf{C}_{n+1}^{e d}= & \frac{\mathbf{C}^{e}}{1+\bar{\mu}_{n}}\left(1-\bar{\gamma}_{n+1} \frac{1}{\left(1+\bar{\mu}_{n}\right)\left\|\boldsymbol{\sigma}_{n+1}^{\text {trial }}\right\|_{\mathbf{D}^{e}}}\right) \\
& +\frac{1}{\left(1+\bar{\mu}_{n}\right)^{2}}\left(\frac{\bar{\gamma}_{n+1}}{\left\|\boldsymbol{\sigma}_{n+1}^{\text {trial }}\right\|_{\mathbf{D}^{e}}}-\frac{1}{\frac{1}{1+\bar{\mu}_{n}}+\frac{\bar{K}}{E}}\right) \mathbf{N}_{n+1}^{\text {trial }} \otimes \mathbf{N}_{n+1}^{\text {trial }}
\end{aligned}
$$

where $\mathbf{N}_{\mathrm{n}+1}^{\text {trial }}=\frac{\boldsymbol{\sigma}_{\mathrm{n}+1}^{\text {trial }}}{\left\|\boldsymbol{\sigma}_{\mathrm{n}+1}^{\text {trial }}\right\|_{\mathbf{p}}}$.

Finally, we carry out the last computation in the local phase at the converged value of internal variables in the sense of the number of active surfaces to set the elastodamage tangent modulus for the next step, according to:

$\mathbf{C}^{e d}=\frac{\partial \mathbf{t}_{\Gamma_{s, n+1}}}{\partial \overline{\mathbf{u}}_{n+1}}=\left\{\begin{array}{l}\overline{\overline{\mathbf{Q}}}_{n}^{-1}-\sum_{i, j=1}^{2}\left[\mathbf{G}_{i j, n+1}\right]^{-1}\left(\overline{\overline{\mathbf{Q}}}_{n}^{-1} \frac{\partial \bar{\Phi}_{i}}{\partial \mathbf{t}_{\Gamma_{s, n+1}}}\right) \otimes\left(\overline{\overline{\mathbf{Q}}}_{n}^{-1} \frac{\partial \bar{\Phi}_{i}}{\partial \mathbf{t}_{\Gamma_{s, n+1}}}\right) \\ \overline{\overline{\mathbf{Q}}}_{n}^{-1}-\frac{\partial \bar{\Phi}_{i}}{\frac{1}{\partial \boldsymbol{t}_{\Gamma_{s, n+1}}} \cdot \overline{\mathbf{Q}}_{n}^{-1} \frac{\partial \bar{\Phi}_{i}}{\partial \boldsymbol{t}_{\Gamma_{s, n+1}}}}\left(\overline{\overline{\mathbf{Q}}}_{n}^{-1} \frac{\partial \bar{\Phi}_{i}}{\partial \mathbf{t}_{\Gamma_{s, n+1}}}\right) \otimes\left(\overline{\overline{\mathbf{Q}}}_{n}^{-1} \frac{\partial \bar{\Phi}_{i}}{\partial \mathbf{t}_{\Gamma_{s, n+1}}}\right)\end{array}\right.$

Having converged with local computation to the final values of internal variables, we turn back to the global phase in order to provide new iterative values of nodal displacements. In this phase, all numerical simulations consider in particular the implicit Newmark scheme with the following residual equations established by applying incompatible mode method (see [17] or [26]) at the end of the time step $t_{n+1}$ and iteration $i$ :

$$
\begin{cases}\mathbf{r}_{n+1}^{(e),(i)}=\mathbb{A}_{e=1}^{n_{e l}}\left[\mathbf{f}_{e x t, n+1}^{(e),(i)}-\mathbf{f}_{i n t, n+1}^{(e),(i)}\right]-M \mathbf{a}_{n+1}^{(e),(i)} & \text { for } \mathbf{x} \in \backslash \Gamma_{s} \\ \mathbf{h}_{n+1}^{(e),(i)}=\int_{\Omega^{e}} \overline{\mathbf{G}}_{\boldsymbol{v}}^{T} \boldsymbol{\sigma}_{n+1}^{(e),(i)} d^{e}+\int_{\Gamma_{s}} \overline{\overline{\mathbf{G}}}_{\boldsymbol{v}}^{T} \mathbf{t}_{\Gamma_{s}} d_{s} & \text { for } \mathbf{x} \in \Gamma_{s}\end{cases}
$$

where $\mathbf{M}, \mathbf{f}_{\text {ext,n+1}}^{(e),(i)}$ and $\mathbf{f}_{\text {int, } n+1}^{(e),(i)}$ are the element mass matrix, external and internal forces, respectively

$$
\begin{aligned}
& \mathbf{M}=\int_{\Omega^{e}}^{\rho} \mathbf{N}^{T} \mathbf{N} d^{e} \\
& \mathbf{f}_{e x t, n+1}^{(e),(i)}=\int_{\Omega^{e}} \mathbf{N b \mathbf { N } ^ { T }} d^{e}+\left[\mathbf{N}^{T} \bar{t}\right]_{\Gamma_{\sigma}} \\
& \mathbf{f}_{i n t, n+1}^{(e),(i)}=\int_{\Omega^{e}} \mathbf{B}^{T} \boldsymbol{\sigma}_{n+1}^{(e),(i)} d^{e}
\end{aligned}
$$

By taking into account interpolations described in the previous section along with using Newton-Raphson method, finally we obtain linearized form of the system of equilibrium equations in (53), according to:

$$
\left[\begin{array}{cc}
A_{e=1}^{n_{e l}} \hat{\mathbf{K}}^{(e)} & A_{e=1}^{n_{e l}} \mathbf{F}^{(e)} \\
\mathbf{F}^{(e), T} & \mathbf{H}^{(e)}
\end{array}\right]_{n+1}^{i}\left(\begin{array}{c}
\Delta \mathbf{d}_{n+1}^{(e),(i)} \\
\Delta \overline{\overline{\mathbf{u}}}_{n+1}^{(e),(i)}
\end{array}\right)=\left(\begin{array}{c}
A_{e=1}^{n_{e l}} \mathbf{r}_{n+1}^{(e),(i)} \\
\mathbf{h}_{n+1}^{(e),(i)}
\end{array}\right)
$$

in which the parts of element stiffness matrix are as follows:

$$
\begin{aligned}
& \hat{\mathbf{K}}_{n+1}^{(e),(i)}=\mathbf{K}_{n+1}^{(e),(i)}+\frac{1}{\beta(\Delta t)^{2}} \mathbf{M} \\
& \mathbf{K}_{n+1}^{(e),(i)}=\frac{\partial \mathbf{f}_{i n t, n+1}^{(e),(i)}}{\partial \mathbf{d}}=\int_{\Omega^{e}} \mathbf{B}^{T} \mathbf{C}_{n+1}^{e d} \mathbf{B} d \Omega^{e} \\
& \mathbf{F}_{n+1}^{(e),(i)}=\frac{\partial \mathbf{f}_{i n t, n+1}^{(e),(i)}}{\partial \overline{\overline{\mathbf{u}}}}=\int_{\Omega^{e}} \mathbf{B}^{T} \mathbf{C}_{n+1}^{e d} \overline{\mathbf{G}}_{r} d \Omega^{e} \\
& \mathbf{F}_{n+1}^{(e),(i), T}=\frac{\partial \mathbf{h}_{n+1}^{(e),(i)}}{\partial \mathbf{d}}=\int_{\Omega^{e}} \overline{\mathbf{G}}_{v}^{T} \mathbf{C}_{n+1}^{e d} \mathbf{B} d \Omega^{e}+\int_{\Gamma_{s}} \overline{\overline{\mathbf{G}}}_{v}^{T} \frac{\partial \mathbf{t}_{\Gamma_{s}}}{\partial \mathbf{d}} d \Gamma_{s}=\int_{\Omega^{e}} \overline{\mathbf{G}}_{v}^{T} \mathbf{C}_{n+1}^{e d} \mathbf{B} d \Omega^{e} \\
& \mathbf{H}_{n+1}^{(e),(i)}=\frac{\partial \mathbf{h}_{n+1}^{(e),(i)}}{\partial \overline{\overline{\mathbf{u}}}}=\int_{\Omega^{e}} \overline{\mathbf{G}}_{v}^{T} \mathbf{C}_{n+1}^{e d} \overline{\mathbf{G}}_{r} d \Omega^{e}+\int_{\Gamma_{s}} \overline{\overline{\mathbf{G}}}_{v}^{T} \frac{\partial \mathbf{t}_{\Gamma_{s}}}{\partial \overline{\overline{\mathbf{u}}}} d \Gamma_{s}
\end{aligned}
$$

Exploting the static condensation at the element level of the second equation, the system (55) is reduced to:

$\mathbb{A}_{e=1}^{n_{e l}}\left(\mathbf{K}_{e f f, n+1}^{(e),(i)} \Delta \mathbf{d}_{n+1}^{(e),(i)}\right)=\mathbb{A}_{e=1}^{n_{e l}} \mathbf{r}_{e f f, n+1}^{(e),(i)}$

where $\mathbf{K}_{\text {eff, }, n+1}^{(e),(i)}, \mathbf{r}_{\text {eff }, n+1}^{(e),(i)}$ are respectively the effective stiffness matrix and effective residual of element.

$$
\begin{aligned}
& \mathbf{K}_{e f f, n+1}^{(e),(i)}=\hat{\mathbf{K}}_{n+1}^{(e),(i)}-\mathbf{F}_{n+1}^{(e),(i)}\left(\mathbf{H}_{n+1}^{(e),(i)}\right)^{-1} \mathbf{F}_{n+1}^{(e),(i), T} \\
& \mathbf{r}_{e f f, n+1}^{(e),(i)}=\mathbf{r}_{n+1}^{(e),(i)}-\mathbf{F}_{n+1}^{(e),(i)}\left(\mathbf{H}_{n+1}^{(e),(i)}\right)^{-1} \mathbf{h}_{n+1}^{(e),(i)}
\end{aligned}
$$

\section{Numerical simulations}

This section presents the results obtained from several numerical tests designed to evaluate and illustrate the performance of the proposed anisotropic damage model. In all examples, the plane strain hypothesis is imposed, the time-dependency of the application of loads is linear increase in time and there is no artificial damping in the simulations. GMSH software [10] is used to generate meshes with constant strain triangle (CST) elements. In the finite element framework, all computations are implemented by a research version of the computer program FEAP, developed by Taylor [29].

\subsection{Simple tension test}

The first test problem is the simple tension in which a rectangular strip with a length of $200 \mathrm{~mm}$, a width equal to $100 \mathrm{~mm}$ and a unit thickness is subjected to homogenous displacementcontrolled tension applied at the right free-end. The boundary conditions and three different finite element meshes employed in computations are presented in Fig. 5. In each mesh, there is a slightly weakened element (shaded area in mesh) in order to better control the macro-crack creation. The set of material properties is given in Table 1.

We see that the computed macro-cracks indicated in Fig. 6 are originated from weakened elements of specimens and then go through the center of neighboring elements in the direction perpendicular to the principal stress at the time when the chosen damage threshold value is reached. Regardless of fineness or 


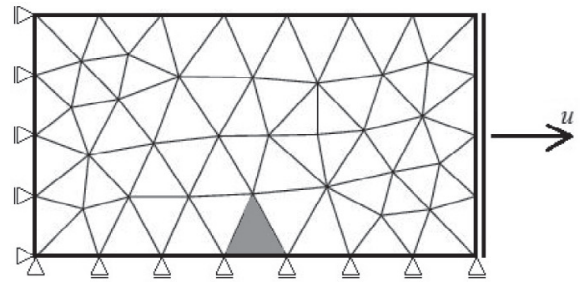

(a) Coarse unstructured mesh (70 elements)

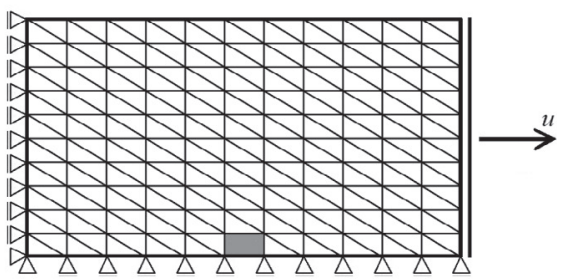

(b) Fine structured mesh (220 elements)

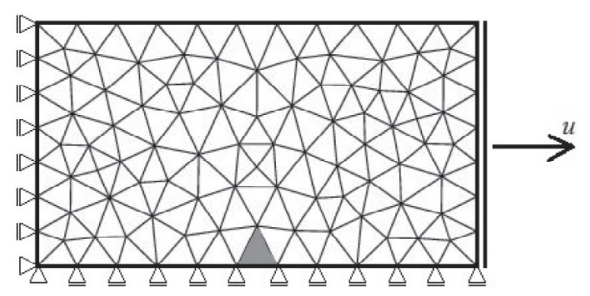

(c) Fine unstructured mesh (182 elements)

Fig. 5. Finite element model and boundary conditions.

Table 1

Material properties for the simple tension test.

\begin{tabular}{ll} 
Continuous model & \\
Young modulus & $38 \mathrm{GPa}$ \\
Poisson's coefficient & 0.18 \\
Density mass & $2600 \mathrm{~kg} / \mathrm{m}^{3}$ \\
$\bar{\sigma}_{f}$ & $2 \mathrm{Mpa}$ \\
$\bar{K}$ & $1,000 \mathrm{MPa}$ \\
Discrete model & \\
$\bar{\sigma}_{f}$ & $2.55 \mathrm{MPa}$ \\
& $2.35 \mathrm{MPa}$ (weakened element) \\
$\overline{\bar{\sigma}}_{s} / \overline{\bar{\sigma}}_{f}$ & 0.3 \\
$\overline{\bar{\beta}}$ & $25.5 \mathrm{MPa} / \mathrm{mm}$ \\
\hline
\end{tabular}

coarseness, two structured and unstructured types of mesh give very different predictions for local response features. Namely, the latter kind cannot produce the macro-crack pattern as a straight

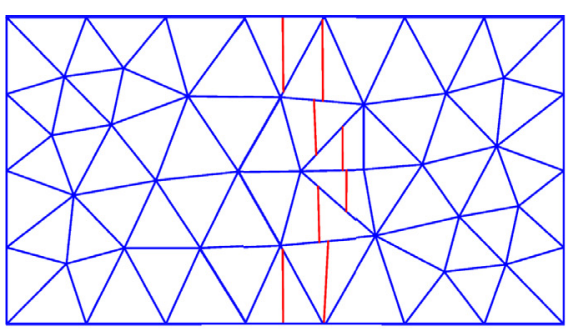

(a) Coarse unstructured mesh line as obtained in the former kind. As for Fig. 7, the obtained results point out that unlike static simulations, where the diagram between load and imposed displacement is exactly the same for all meshes, in dynamic element tests the global response computed for meshes in terms of the load versus displacement curve is dissimilar. The reason for this difference is that the solution is affected by the inertia effect which is always present in dynamic problems, leading to different wave frequencies for various meshes.

\subsection{Brazilian-like semicircular disc test}

In this example, we present the simulated results of the Brazilian-like semicircular disc test. Table 2 shows material properties of the specimen. The computational model for simulations is described in Fig. 8 where a semicircular disc with $10 \mathrm{~mm}$ in diameter and a unit thickness is indirectly applied homogeneous downward displacements through a rectangular block put over it, or other words, this test is carried out under displacement control

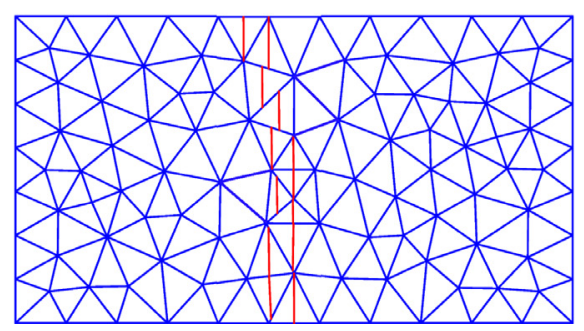

(c) Fine unstructured mesh

Fig. 6. Crack path at the end of the computation for structured and unstructured meshes. 


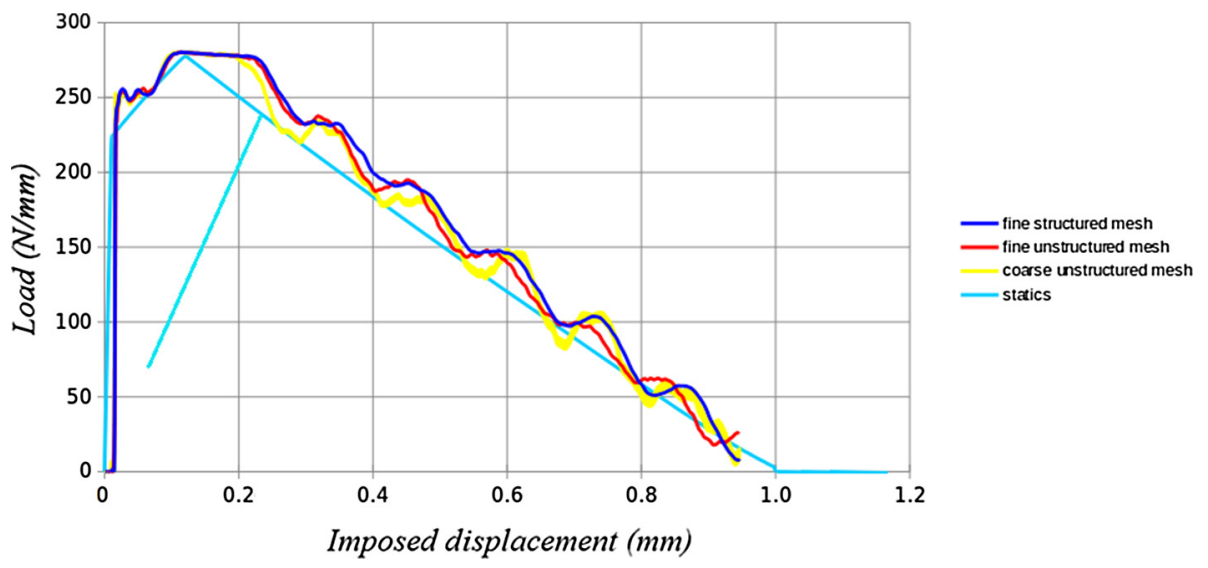

Fig. 7. Load-imposed displacement diagram for three different discretizations.

Table 2

Material properties of the specimen.

$\begin{array}{ll}\text { Continuous model } & \\ \text { Young modulus } & \\ & \\ \text { Poisson's coefficient } & 75 \mathrm{GPa} \text { (semi-disc) } \\ \text { Density mass } & 0.18 \\ & 2600 \mathrm{~kg} / \mathrm{m}^{3} \text { (semi-disc) } \\ \bar{\sigma}_{f} & 3000 \mathrm{~kg} / \mathrm{m}^{3} \text { (rectangular block) } \\ \bar{K} & 2 \mathrm{Mpa} \\ \text { Discrete model } & 1000 \mathrm{MPa} \\ \overline{\bar{\sigma}}_{f} & \\ & \\ \overline{\bar{\sigma}}_{s} / \overline{\bar{\sigma}}_{f} & 2.55 \mathrm{MPa} \\ \overline{\bar{\beta}} & 2.35 \mathrm{MPa} \text { (weakened element) } \\ \end{array}$

with note that precise boundary condition between the rectangular block and the semicircular disc is unilateral contact with no friction (e.g. see [14], Ch. 5). A coarse mesh with 202 elements and a fine mesh with 682 elements are used for the computation. In each mesh, a single element is slightly weakened (red area in mesh) to better orientate the macro-crack occurrence.

From results in Fig. 9 in which crack opening at the end of the computation for both meshes is indicated, it can be seen that a similar crack path, which is predicted for two different discretizations, agrees quite well with the experimental results. Namely, from the experimental point of view, fracture originates from the tips of microcracks lying perpendicular to the direction of principal stress and should be located at the center of the semicircular disc. The resulting crack would propagate in the loading direction, and the specimen would eventually split into two halves along the

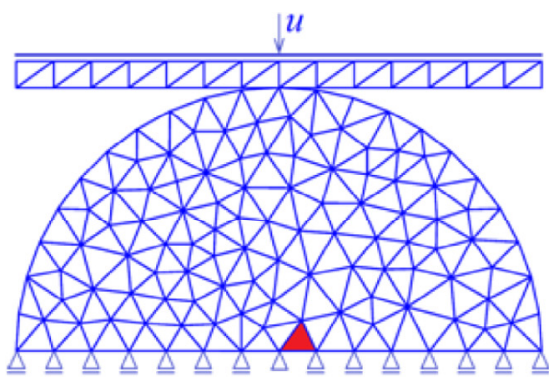

(a) Coarse mesh (202 elements)

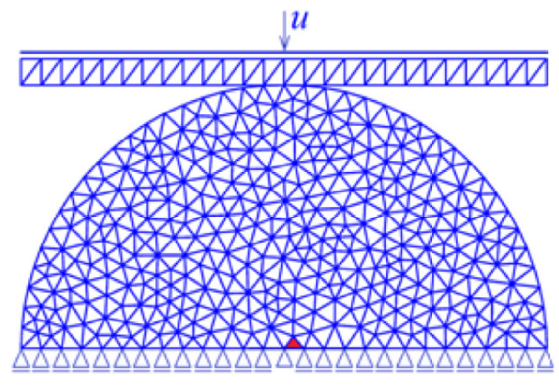

(b) Fine mesh (682 elements)

Fig. 8. Computational model for Brazilian-like semicircular disc test.

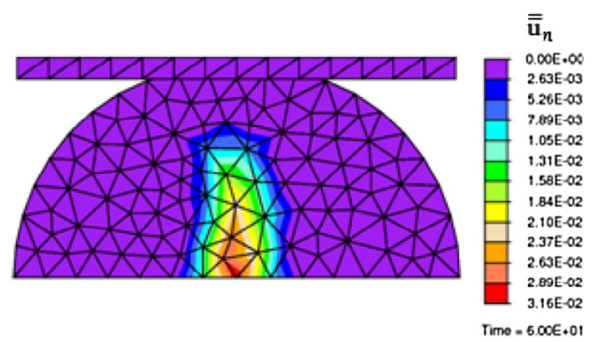

(a) Coarse mesh

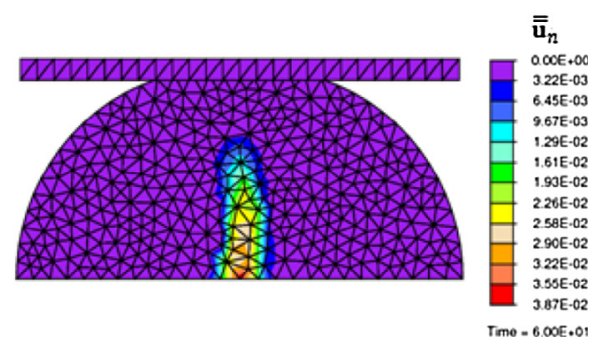

(b) Fine mesh

Fig. 9. Spread of the FPZ (Fracture process zone) at the end of the computation for the coarse and the fine mesh. 


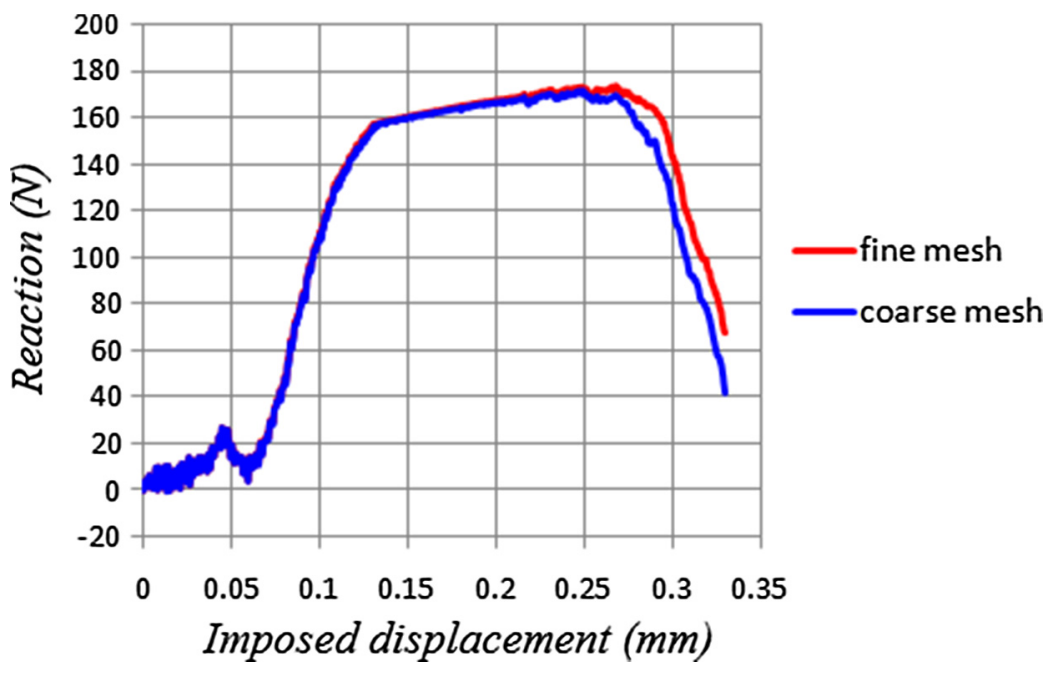

Fig. 10. Reaction in terms of displacement.

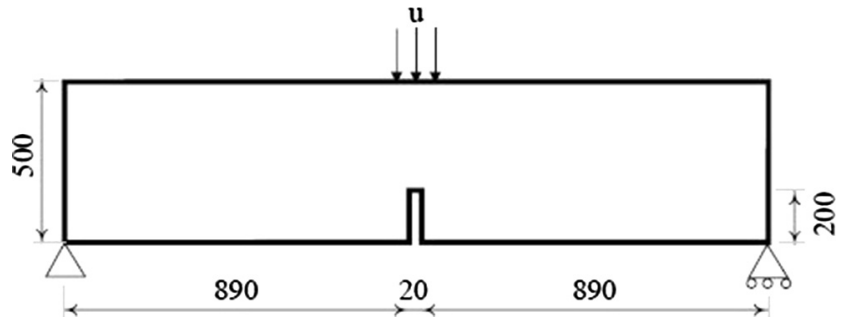

Fig. 11. Geometric characteristics (in $\mathrm{mm}$ ) and boundary conditions of the notched specimen.

Table 3

Material properties for the three-point bending test.

\begin{tabular}{ll}
\hline Continuous model & \\
Young modulus & $38 \mathrm{GPa}$ \\
Poisson's coefficient & 0.1 \\
Density mass & $2600 \mathrm{~kg} / \mathrm{m}^{3}$ \\
$\bar{\sigma}_{f}$ & $2.2 \mathrm{Mpa}$ \\
$\bar{K}$ & $1000 \mathrm{MPa}$ \\
Discrete model & \\
$\overline{\bar{\sigma}}_{f}$ & $2.35 \mathrm{MPa}$ \\
$\overline{\bar{\beta}}$ & $23.5 \mathrm{MPa} / \mathrm{mm}$ \\
$\overline{\bar{\sigma}}_{s}$ & $0.235 \mathrm{MPa}$ \\
\hline
\end{tabular}

compressive diametral line. Fig. 10 shows reaction versus imposed displacement relation. Simulated results point out a little difference between two meshes.

\subsection{Three-point bending test}

We consider next the three-point bending test of a notched concrete beam. Fig. 11 describes the geometry of the specimen, the boundary conditions and the loading in which downward displacements are imposed at center top of the beam in order to ensure this element test is performed under displacement control. The chosen values of material parameters are given in Table 3. Two different unstructured meshes shown in Fig. 12 are exploited in the computational procedure.

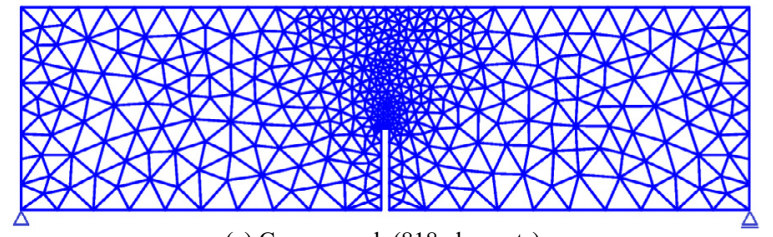

(a) Coarse mesh (818 elements)

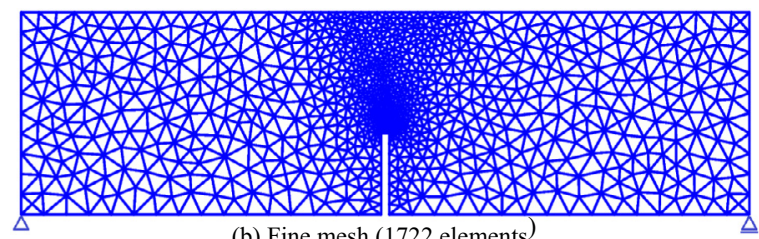

(b) Fine mesh (1722 elements

Fig. 12. Two kinds of the finite element mesh used for computation.

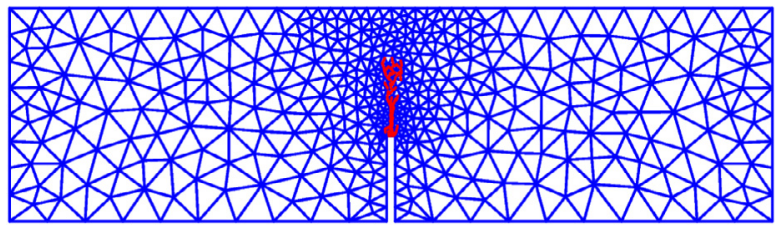

(a) Coarse mesh

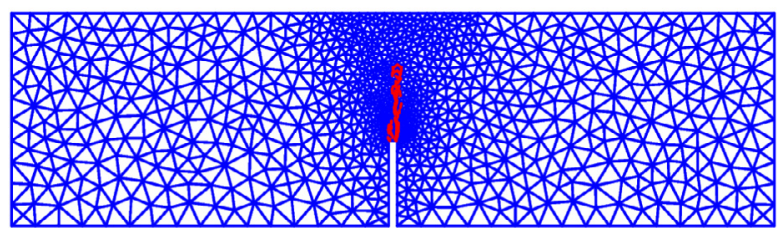

(b) Fine mesh

Fig. 13. Crack path at the end of the computation for the coarse and the fine mesh

As indicated in Fig. 13, for both meshes the discontinuity line starts at the notch and propagate perpendicularly to the length of the beam. This tendency of development of macro-cracks is identical to that of experimental results (see [23]). Turning to the 


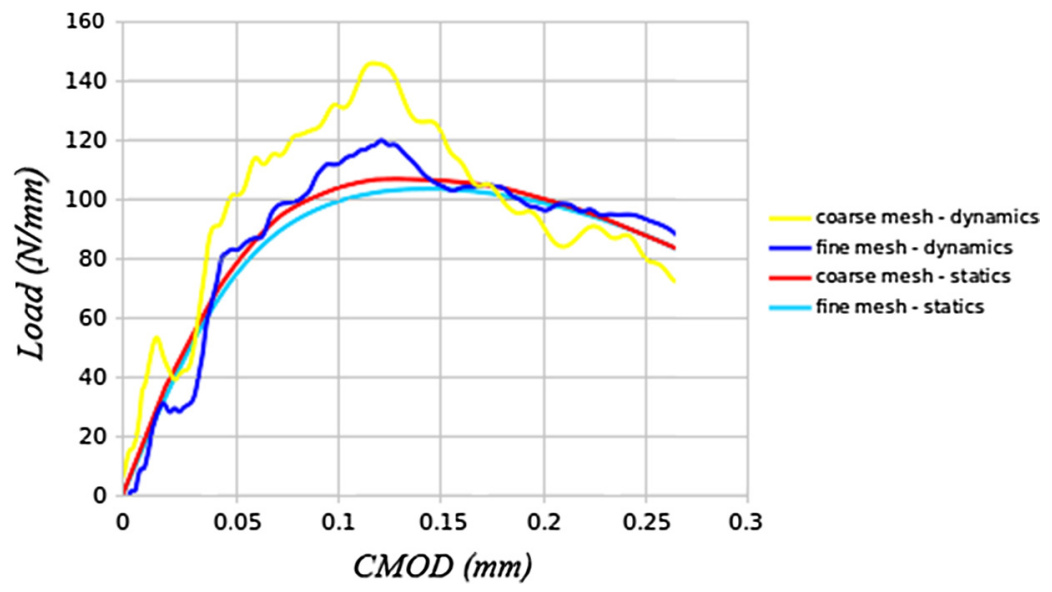

Fig. 14. Measured load-crack mouth opening displacement (CMOD) curve.

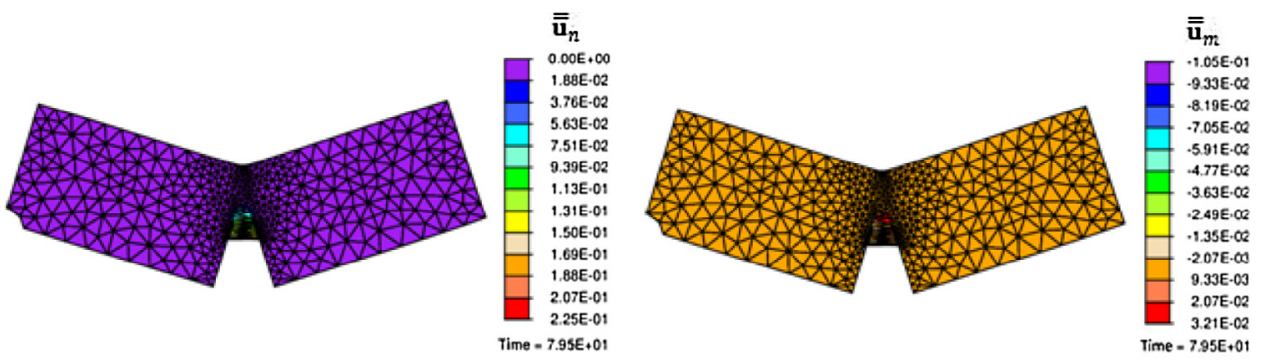

(a) Coarse mesh

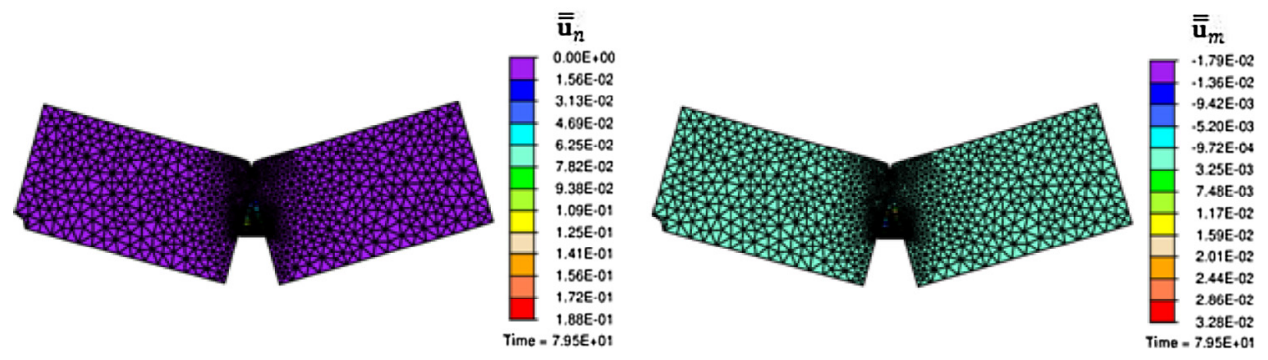

(b) Fine mesh

Fig. 15. Crack opening $\overline{\overline{\mathbf{u}}}_{n}$ and sliding $\overline{\overline{\mathbf{u}}}_{m}$ at the end of the computation for two different discretizations.

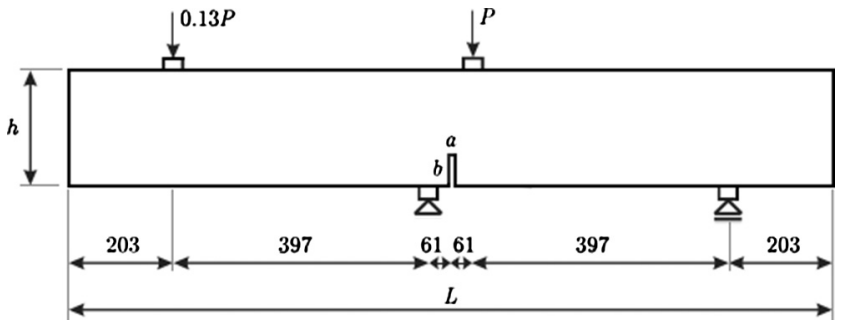

Fig. 16. Notched specimen: geometric characteristics (in $\mathrm{mm}$ ) with $\mathrm{L}=1322 \mathrm{~mm}$, $\mathrm{h}=306 \mathrm{~mm}, \mathrm{a}=14 \mathrm{~mm}, \mathrm{~b}=82 \mathrm{~mm}$ and boundary conditions.

Fig. 14 which plots measured load in terms of crack mouth opening displacement (CMOD), we find once again that even though two curves does not totally coincide due to inertia effect the global response computed for two different finite element meshes has
Table 4

Material properties of the anisotropic damage model in the four-point bending test.

\begin{tabular}{ll}
\hline Continuous model & \\
Young modulus & $28.8 \mathrm{GPa}$ \\
Poisson's coefficient & 0.18 \\
Density mass & $2600 \mathrm{~kg} / \mathrm{m}^{3}$ \\
$\bar{\sigma}_{f}$ & $2.6 \mathrm{Mpa}$ \\
$\bar{K}$ & $1000 \mathrm{MPa}$ \\
Discrete model & \\
$\overline{\bar{\sigma}}_{f}$ & $2.8 \mathrm{MPa}$ \\
$\overline{\bar{\sigma}}_{s} / \overline{\bar{\sigma}}_{f}$ & 0.1 \\
$\overline{\bar{\beta}}$ & $28 \mathrm{MPa} / \mathrm{mm}$ \\
\hline
\end{tabular}

quite similar trends, or more precisely, absolute vertical or relative horizontal evolution of displacement as a function of applied loading. It is also important to note that the same propensities keep 


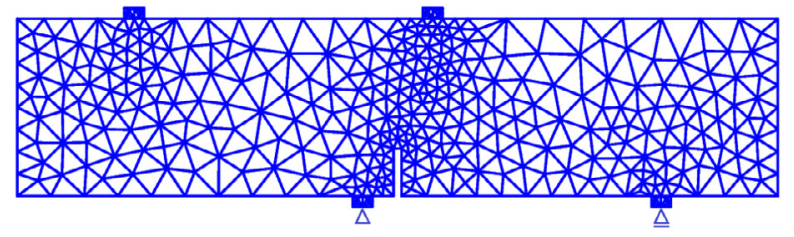

(a) Coarse mesh (723 elements)

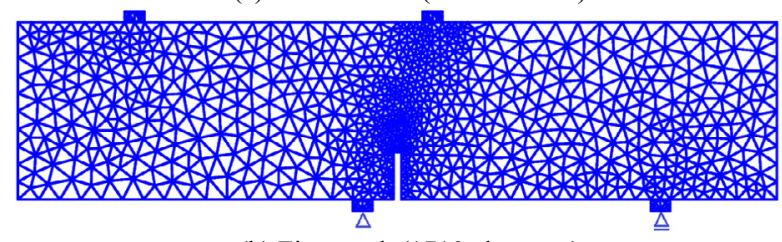

(b) Fine mesh (1710 elements)

Fig. 17. Finite element meshes used for computation.

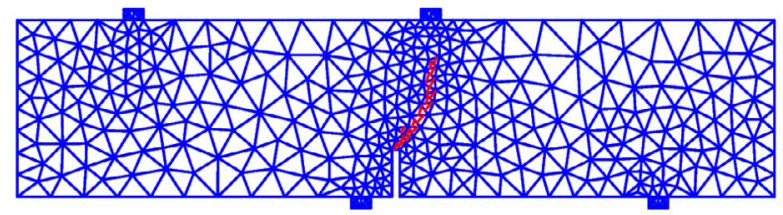

(a) Coarse mesh

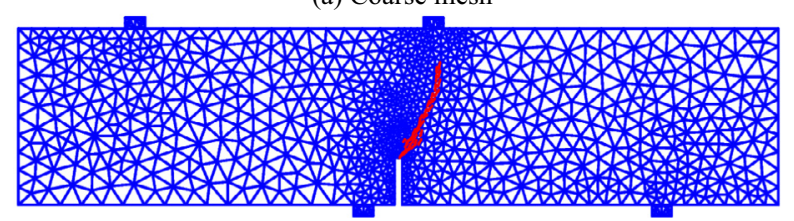

(b) Fine mesh

Fig. 18. Crack path at the end of the computation for two types of meshes.

being observed for the internal variable (a prolongation of CMOD) in Fig. 15 where crack opening $\overline{\mathbf{u}}_{n}$ and crack sliding $\overline{\overline{\mathbf{u}}}_{m}$ at the final deformed configurations (scaled 250 times) for two different dis- cretizations are shown. The dynamics framework increase the model robustness. As illustration, the total number of iteration is reduced in half $(23,841$ versus 43,466 by using quasi-static approach) until 1851th step when quasi-static model can no longer converge. By adding the enhancement to the discontinuity interface law in terms of rate sensitivity, for the present case of quasi-static testing it makes not much difference - the response is slightly increased, while the number iterations remain the same.

\subsection{Four-point bending test}

To end this section, we now study the four-point bending of a plain concrete notched beam. Fig. 16 depicts the boundary conditions, the loading and the geometry of the specimen with note that four blocks located between the applied load and the concrete beam as well as between the supports and the concrete beam are steel caps having Young's modulus $E=288 \mathrm{GPa}$, density mass of $7830 \mathrm{~kg} / \mathrm{m}^{3}$ and Poisson's coefficient equal to 0.18 . In addition, contrary to the three-point bending test, in which the load is applied directly through imposing the displacement, in this case, loads $P$ and $0.13 \mathrm{P}$ are imposed on the steel caps at the top surface of the beam. Material parameters of the concrete beam are given in Table 4. Two different unstructured meshes, the coarse mesh with 723 elements and fine mesh with 1710 elements (Fig. 17) are considered for simulations.

According to the results in Fig. 18, we can see that similar to the experimental results (see [3]) the crack in both meshes initiates at the top right corner of the notch, propagates up and to the right. Fig. 19 shows the curve obtained for load versus crack mouth opening displacement (CMOD). The simulated results indicate a quite identical tendency in computed paths for the coarse and the fine mesh, namely: the evolution of displacement as a function of applied loading even though there still exists a small difference in obtained values of load and displacement. Finally, we can find that the local results in terms of crack opening and crack sliding presented in Fig. 20 remain quite similar for two types of mesh.

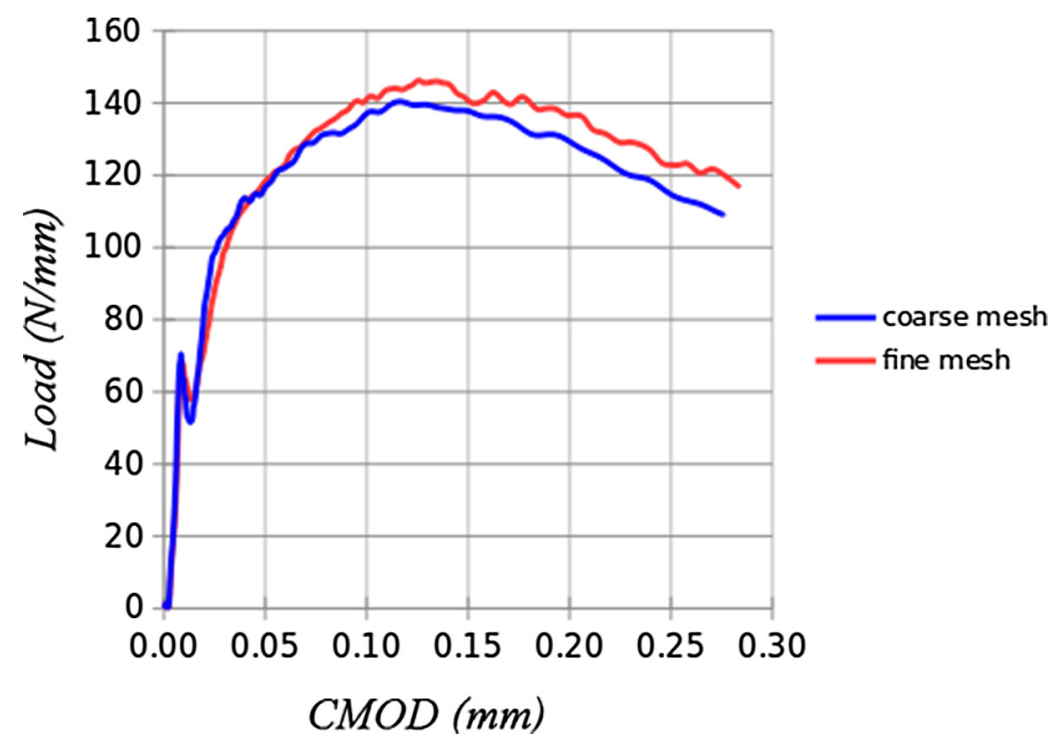

Fig. 19. Load-crack mouth opening displacement (CMOD) response. 

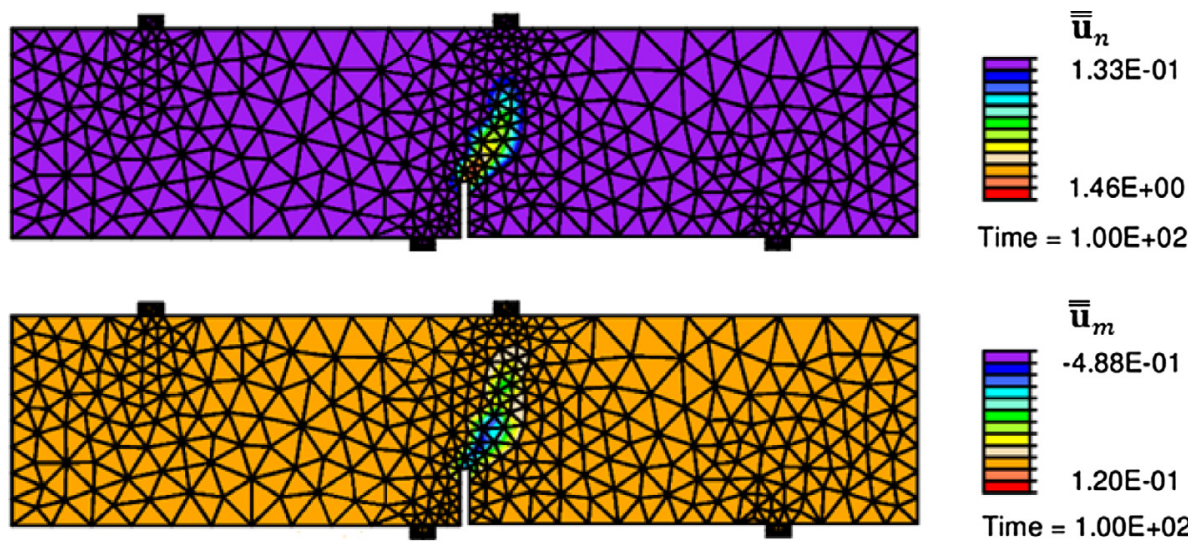

Time $=1.00 \mathrm{E}+02$

(a) Coarse mesh
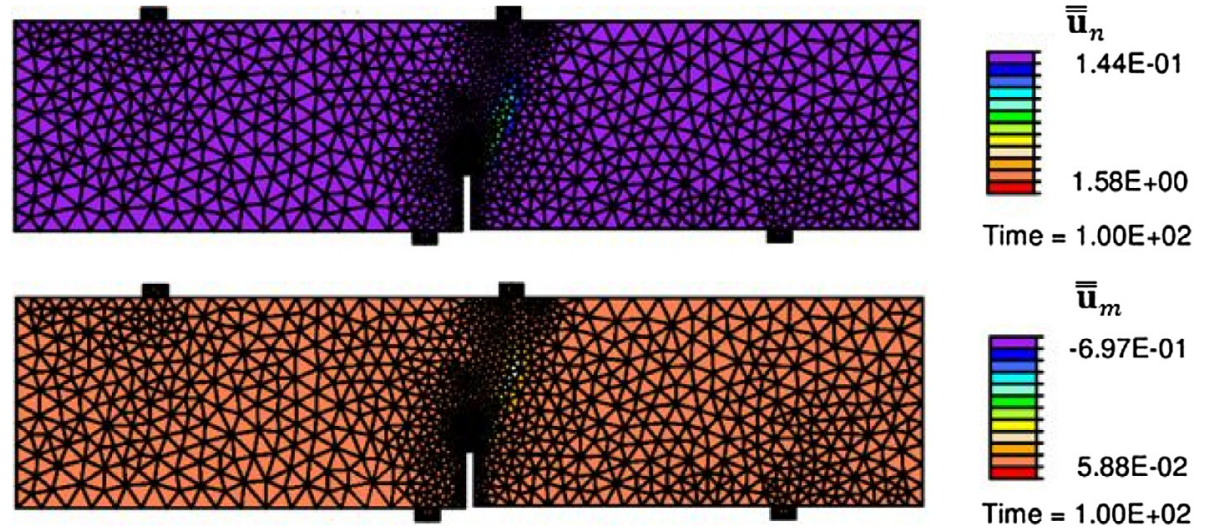

(b) Fine mesh

Fig. 20. Crack opening $\overline{\overline{\mathbf{u}}}_{n}$ and sliding $\overline{\overline{\mathbf{u}}}_{m}$ at the end of the computation for the coarse and the fine mesh.

\section{Conclusions}

In this work, we have presented a two-dimensional multisurface anisotropic damage model combining mechanisms of continuum damage and embedded strong discontinuity in the computational framework of nonlinear dynamics. Thanks to this combination, the proposed model is capable of representing the localized failure of massive structure in dynamics framework, resulting in quite well prediction for propagation direction of the crack. In addition, coupling two failure modes: mode I and mode II in the right way leads the model to desirable convergence for large computations in which a lot of different phenomena happen at the same time. As a consequence, the model proposed herein can be used in simulations for different heterogeneous materials where localized discontinuities have a strong influence on the material's behavior. Finally, the proposed dynamics framework renders the computations more robust, with the possibility to include eventual inertia effect for rapid failure in localization phase.

\section{Acknowledgments}

This work was supported by the excellence scholarship from Vietnamese Ministry of Education and Training and funding of Chaire de Mécanique Picardie. This financial support is gratefully acknowledged.

\section{References}

[1] Alfaiate J, Sluys LJ. A discrete strong embedded discontinuity approach. Eng Fract Mech 2002:69:661-86.

[2] Alfaiate J, Wells GN, Sluys LJ. On the use of embedded discontinuity elements with crack path continuity for mode-I and mixed-mode fracture. Eng Fract Mech 2002:69:661-86.

[3] Arrea M, Ingraffea AR. Mixed-mode crack propagation in mortar and concrete Report No. 81-13. Ithaca (NY): Department of Structural Engineering, Cornell University; 1982

[4] Armero F. Localized anisotropic damage of brittle materials. In: Onate E, Owen DRJ, Hinton E, editors. Computational plasticity. Barcelona: Fundamentals and Applications, CIMNE; 1997. p. 635-40.

[5] Armero F, Linder C. Numerical simulation of dynamic fracture using finite elements with embedded discontinuities. Int J Fract 2009;160:119-41.

[6] Armero F, Garikipati K. Recent advances in the analysis and numerical simulation of strain localization in inelastic solids. In: Owen E, Onate DRJ, Hinton E, editors. Proceedings of computational plasticity IV. Barcelona: CIMNE; 1995. p. 547-61.

[7] Bazant ZP, Belytschko T, Chang TP. Continuum theory for strain softening. J Eng Mech 1984:110(12):1666-91.

[8] Belytschko T, Black T. Elastic crack growth in finite elements with minimal remeshing. Int J Numer Meth Eng 1999;45:601-20.

[9] Brancherie D, Ibrahimbegovic A. Novel anisotropic continuum-discrete damage model capable of representing localized failure of massive structures: part I: theoretical formulation and numerical implementation. Eng Comput 2009;26:100-27.

[10] Geuzaine C, Remacle JF. Gmsh: a three-dimensional finite element mesh generator with built-in pre- and postprocessing facilities. Int J Numer Meth Eng 2009;11:1309-31.

[11] Dujc J, Brank B, Ibrahimbegovic A. Stress-hybrid quadrilateral finite element with embedded strong discontinuity for failure analysis of plane stress solids. Int J Numer Meth Eng 2013;94:1075-98.

[12] Hillerborg A, Modeer M, Petersson PE. Analysis of crack formation and crack growth in concrete by means of fracture mechanics and finite elements. Cem Concr Res 1976;6:773-82. 
26

X.N. Do et al./Computers and Structures 183 (2017) 14-26

[13] Huespe AE, Oliver J, Sanchez PJ, Blanco S, Sonzogni V. Strong discontinuity approach in dynamic fracture simulations. Mecánica Computational 2006;25:1997-2018.

[14] Ibrahimbegovic A. Nonlinear solid mechanics: theoretical formulations and finite element solution methods. Berlin (Germany): Springer; 2009.

[15] Ibrahimbegovic A, Kozar I. Non-linear Wilson's brick element for finite elastic deformations of three-dimensional solids. Common Numer Methods Eng 1995; 11:655-64.

[16] Ibrahimbegovic A, Melnyk S. Embedded discontinuity finite element method for modeling of localized failure in heterogeneous materials with structured mesh: an alternative to extended finite element method. Comput Mech 2007;40:149-55.

[17] Ibrahimbegovic A, Wilson EL. A modified method of incompatible modes. Common App Numer Meth 1991;7:187-94.

[18] Lemaitre J. A course on damage mechanics. New York (NY): Springer; 1992.

[19] Needleman A. Material rate dependence and mesh sensitivity in localization problems. Comput Methods App Mech Eng 1988;63:69-85.

[20] Oliver J. On the discrete constitutive models induced by strong discontinuity kinematics and continuum constitutive equations. Int J Solids Struct 2000;37:7207-29.

[21] Ortiz M. A constitutive theory for inelastic behavior of concrete. Mech Mater 1985;4:67-93.

[22] Ortiz M, Leroy Y, Needleman A. A finite element method for localized failure analysis. Comput Methods Appl Mech Eng 1987;61:189-214.
[23] Peterson PE. Crack growth and development of fracture zones in plain concrete and similar materials Report No. TVBM-1006. Land (Sweden): Division of Building Materials, University of Land; 1981.

[24] Radulovic R, Bruhns OT, Mosler J. Effective 3D failure simulations by combining the advantages of embedded Strong Discontinuity Approaches and classical interface elements. Eng Fact Mech 2011;78:2470-85.

[25] Rots JG, Nauta P, Kusters G, Blaauwendraa T. Smeared crack approach and fracture localization in concrete. Heron 1985;30(1):1-48.

[26] Sims JC, Rifai MS. A class of mixed assumed strain methods and the method of incompatible modes. Int J Numer Meth Eng 1990;29:1595-638.

[27] Sumo JC, Oliver J, Armero F. An analysis of strong discontinuity induced by strain softening solutions in rate-independent solids. J Compute Mech 1993;12:277-96.

[28] Sluys LJ. Modelling of crack propagation with embedded discontinuity elements. In: Mihashiand $\mathrm{H}$, Rokugo $\mathrm{K}$, editors. Fracture mechanics of concrete structures, FRAMCOS3. Japan: Gifu; 1998. p. 843-60.

[29] Taylor R. FEAP finite element analysis program. University of California: Berkeley <http://www.ce.berkeley.edurlt>.

[30] Wells GN, Sluys LJ. Analysis of slip planes in three-dimensional solids. Comput Methods App Mech Eng 2001;190:3591-606.

[31] Dvorkin EN, Cuitiño AM, Gioia G. Finite elements with displacement interpolated embedded localization lines insensitive to mesh size and distortions. Int J Numer Meth Eng 1990;30:541-64.

[32] Dvorkin EN, Assanelli AP. 2D finite elements with displacement interpolated embedded localization lines: the analysis of fracture in frictional materials. Comput Methods App Mech Eng 1991;90:829-44. 